\title{
28. LATE CENOZOIC ATMOSPHERIC CIRCULATION INTENSITY AND CLIMATIC HISTORY RECORDED BY EOLIAN DEPOSITION IN THE EASTERN EQUATORIAL PACIFIC OCEAN, LEG 138 ${ }^{1}$
}

\author{
Steven A. Hovan ${ }^{2}$
}

\begin{abstract}
Sediments recovered during Ocean Drilling Program (ODP) Leg 138 in the eastern equatorial Pacific Ocean were analyzed for variations in eolian accumulation rate and mean grain-size. Latitudinal and temporal patterns of these parameters showed important changes in the intensity of atmospheric circulation and eolian flux associated with the intertropical convergence zone (ITCZ) and suggested that eolian input parameters could be used to define its paleoposition through time. Modern atmospheric circulation in the equatorial region is weakest in the intertropical convergence zone and increases as the trade winds are approached to the north and south. Thus, the expected spatial pattern of eolian grain size would have the finest material deposited beneath the ITCZ and a coarsening of material in both directions away from this zone. Sediments from ODP Leg 138 show this pattern for much of the Pleistocene and Pliocene but, prior to about $4 \mathrm{Ma}$, begin to lose the northern coarse component suggesting that the ITCZ was located north of its present position during the late Miocene. Eolian flux records also show a latitudinal pattern of deposition associated with the position of the ITCZ that, similar to eolian grain-size variability, suggests a more northerly position of the ITCZ during the late Miocene. Overall, the regional input of eolian material to the equatorial Pacific has decreased throughout the late Neogene. This reduction in eolian input reflects climatic changes to relatively wetter conditions in the continental eolian source regions beginning during the late Pliocene.
\end{abstract}

\section{INTRODUCTION}

The equatorial oceans are greatly influenced by changes in Earth's zlimate system. The biology, chemistry, and sedimentation in this region are closely related to changes in oceanic circulation, which varies primarily in response to atmospheric circulation. Atmospheric circulation, in turn, is strongly influenced by hemispherical or global climatic conditions. Sediments from the equatorial Pacific contain multichannel records of both atmospheric and oceanic circulation changes, and therefore provide a unique opportunity to study both the long-term history and interactions between several of these subsystems of climate in the geologic past.

\section{Atmospheric and Oceanic Circulation in the Equatorial Pacific}

In the tropical Pacific, the southeast and northeast trade winds converge north of the geographic equator (Fig. 1). The place where these two wind systems meet is the intertropical convergence zone (ITCZ); it is characterized by light and variable winds and increased rainfall. The relative strength of the northeast and southeast trade winds governs the latitudinal position of the ITCZ and the position and strength of the oceanic surface currents in the equatorial region (Fig. 2). As southern trade winds blow across the equator, the change in direction of the Coriolis effect causes divergence of water away from the equator that is geostrophically balanced by the westward flow of the South Equatorial Current (SEC). Fluctuation in the strength of the SEC follows the seasonal cycle of the strength of the southeast trade winds. During the southern hemisphere winter, when the southeast trade winds are strongest, the SEC is most intense; during the summer, the SEC weakens.

The North Equatorial Current (NEC) lies north of about $10^{\circ} \mathrm{N}$ and is associated with the northeast trade winds (Fig. 1). Unlike the SEC, the intensity of the NEC appears to be more related to the location of

\footnotetext{
'Pisias, N.G., Mayer, L.A., Janecek, T.R., Palmer-Julson, A., and van Andel, T.H. (Eds.), 1995. Proc. ODP, Sci. Results, 138: College Station, TX (Ocean Drilling Program).

${ }^{2}$ Department of Geological Sciences, 1006 C.C. Little Building, University of Michigan, Ann Arbor, MI 48109-1063, U.S.A.
}

the trade winds rather than to their strength (Wyrtki, 1974). During the first half of the year, northeast trade winds are located at their southernmost position and are most intense, yet the NEC is weak. The NEC strengthens during the second half of the year when the tradewind belt is at a more northerly position (about $15^{\circ} \mathrm{N}$ ) and its full strength acts on the NEC.

The Equatorial Counter Current (ECC) is an eastward-flowing current lying between the NEC and SEC. The ECC fluctuates synchronously with the NEC (Wyrtki, 1974). The ECC is strongest during Northern Hemisphere summer, when the ITCZ is at its northerly position and opposing surface trade-wind flow is weaker.

Thus, atmospheric and oceanic circulations are closely linked in the equatorial Pacific. One of the primary goals of Leg 138 (Mayer, Pisias, Janecek, et al., 1992) was to recover a continuous, high-resolution pelagic sedimentary section to examine late Neogene paleoceanographic and paleoclimatic changes in this region (Fig. 3). Many researchers have examined changes in oceanic circulation recorded by chemical and compositional changes in sediments from Leg 138 (e.g., Farrell et al., Hagelberg et al., Mix et al., Pisias et al., and others in this volume). This study focuses on changes in the atmospheric circulation throughout the late Neogene derived from the continental eolian component of these sediments. Together, these studies provide direct and independent records of the long-term relationship between atmospheric and oceanic circulation in the equatorial Pacific.

\section{Eolian Proxy Records: Continental Climate,} Atmospheric Circulation Intensity, and ITCZ Location

The mass flux of eolian material to equatorial Pacific primarily reflects changes in the climatologically controlled supply of dust from the continental source region (Prospero, 1981; Rea et al., 1985; Prospero and Nees, 1986). Arid climates reduce vegetational cover, thereby allowing increased wind erosion and dust input to the atmosphere. Isotopic and trace element studies of eolian sediments suggest that the Americas supply most of the dust material transported by the Southern Hemisphere trade winds in the equatorial Pacific (Nakai et al., 1993). Hence, the regional record of eolian mass flux to this area presumably reflect climatic conditions in the continental source regions of Central America and northern South America. Superimposed 


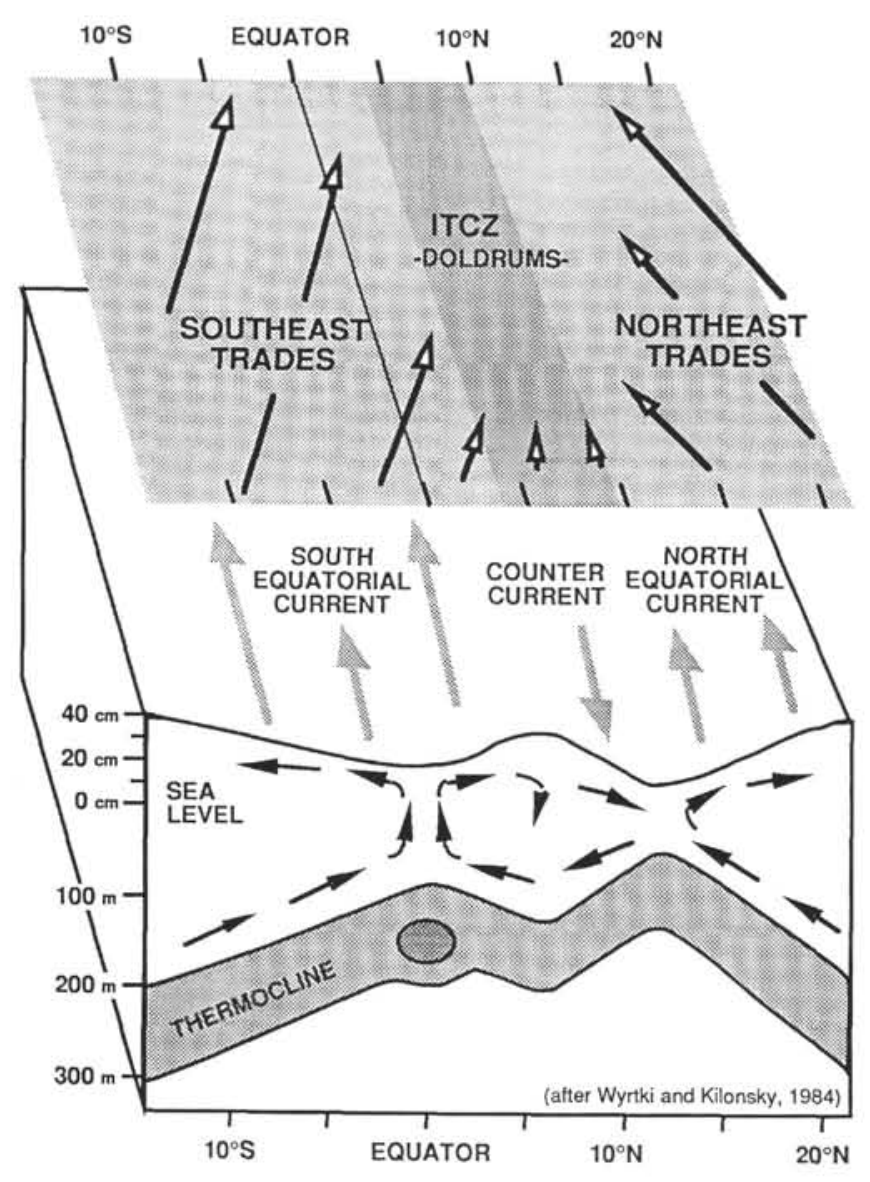

Figure 1. Schematic diagram showing the major features of surface ocean and atmospheric circulation in the eastern equatorial Pacific.

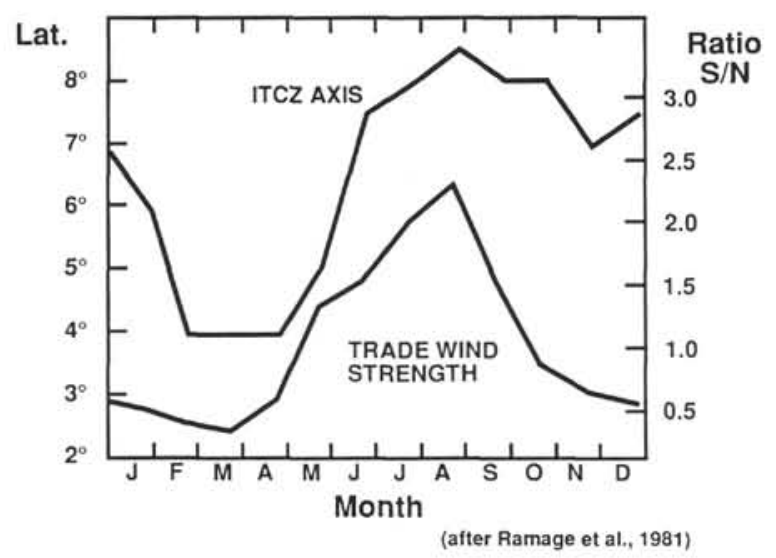

Figure 2. Monthly position of intertropical convergence zone (ITCZ) axis and ratio of South Pacific trade-wind strength to North Pacific trade-wind strength.

upon this climatic control are effects related to atmospheric depositional processes and the rate of precipitation scavenging from the atmosphere. Meteorologic conditions within the ITCZ are characterized by enhanced downward mixing and wet-depositional scavenging of aerosol particles which effectively form a barrier to latitudinal eolian transport (Raemdonck et al., 1986; Rea, 1990). Furthermore, these processes result in more efficient removal of aerosol particles from the atmosphere, which is reflected by increased flux of eolian sediments to the pelagic ocean beneath the ITCZ. Thus, temporal and

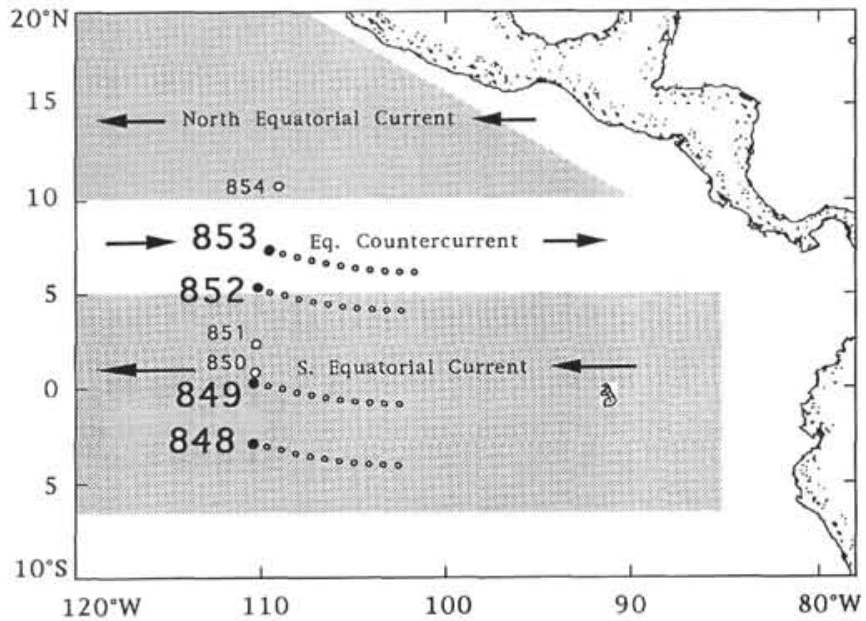

Figure 3. Map showing the location of drill sites along the western transect $\left(110^{\circ} \mathrm{W}\right)$ of Leg 138 . Sites used in this study are denoted by large, boldface lettering and show backtrack locations of sites at successive 1-m.y. intervals based on tectonic reconstructions of Cox and Engebretson, 1985.

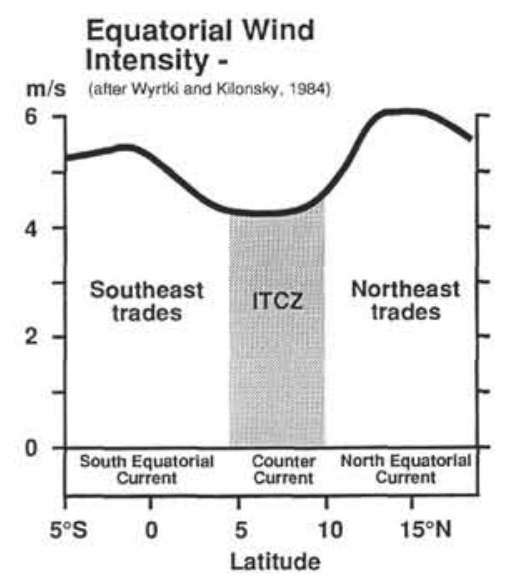

Figure 4. Modern distribution of wind strength in the equatorial Pacific showing less intense winds associated with the ITCZ.

spatial patterns of eolian flux provide a method to monitor changes in regional climatic conditions and to track the latitudinal position of the ITCZ in the equatorial Pacific.

The best records of the atmospheric wind strength have come from measuring grain-size variations in continental mineral grains transported to the ocean by eolian processes (Janecek and Rea, 1985; Rea et al., 1985; Chuey et al., 1987; Pisias and Rea, 1988; Clemens and Prell, 1990). Changes in the intensity of atmospheric circulation are directly reflected in the size distribution of small grains transported in equilibrium with the winds (Gillette et al., 1974; Janecek, 1985). As dust is initially raised from continental source regions, grains are a complex mixture of sizes and shapes. Beyond about $1000 \mathrm{~km}$ from its source region, land and air-based sampling (Gillette et al., 1974; Glaccum and Prospero, 1980) and theoretical calculations (Windom, 1969; Jaenicke, 1979; Schutz et al., 1981) suggest its size distribution changes little. Therefore, temporal variations in the size of eolian particles can be used to reconstruct the history of paleowind intensity. In the equatorial Pacific, the latitudinal pattern of zonal wind intensity and the position of the ITCZ are closely related and show that the intensity of atmospheric circulation is weakest beneath the ITCZ and strengthens to the north and south (Fig. 4). Thus, similar to eolian flux records, temporal and spatial patterns of eolian grain-size parameters can be used to 
monitor changes in the intensity of atmospheric circulation and determine the paleoposition of the ITCZ throughout the geologic past.

\section{ANALYTICAL METHODS}

Sites were selected from the north-south transect of sites near $110^{\circ} \mathrm{W}$ to avoid the regime of fluvial and hemipelagic terrigenous input near the continents and to provide the broadest possible latitudinal distribution (Fig. 3). Data from Site 854 are not included in this study because the mineral fraction isolated from these sediments was severely contaminated with volcanically related material not representative of eolian transport. In addition, in the lower portion of each sequence hydrothermal sediments become a significant sedimentary component and may overwhelm the eolian signal. All other samples contained minimal volcanic contamination.

The eolian fraction of these sediments was isolated using a series of chemical extractions, as outlined by Rea and Janecek (1981). Bulk sediments were treated with acetic acid to remove carbonate components and a buffered sodium citrate and sodium dithionite solution to remove oxides and hydroxides. The procedure was modified to digest the biogenic silica component because many samples contained high concentrations of diatoms that proved difficult to dissolve using the sodium carbonate technique described by Rea and Janecek (1981). After $2 \mathrm{hr}$ of digestion in a $1.5-\mathrm{N}$ sodium hydroxide solution at $85^{\circ} \mathrm{C}$, effectively all of the biogenic silica was removed from the samples. However, because this modified procedure leaches a portion of the crystalline silicate component as well, a correction was applied to mineral component percentage data to compensate for the amount of crystalline silicate dissolved (Fig. 5). This modified procedure does not appear to affect the grain-size distribution significantly. Samples analyzed prior to treatment show nearly identical grain-size distributions to those run after digestion in sodium hydroxide, suggesting that most of the silicate dissolution occurs in the less than $1 \mu \mathrm{m}$ fraction. Mineral percentages estimated using this procedure are precise to within $\pm 0.14 \%$ for samples containing less than $1.0 \%$ mineral component; precision increases with greater mineral concentrations.

The mass flux or accumulation rate of eolian material was calculated by multiplying the eolian percentage by the dry-bulk density (DBD) and linear sedimentation rate (LSR) determined for each sample. Dry-bulk densities were determined from porosity values calculated by measuring the water content of each bulk sample. To minimize desiccation before analysis, sediment samples were stored with a moist tissue in two sealed plastic bags. For samples that did not have bulk

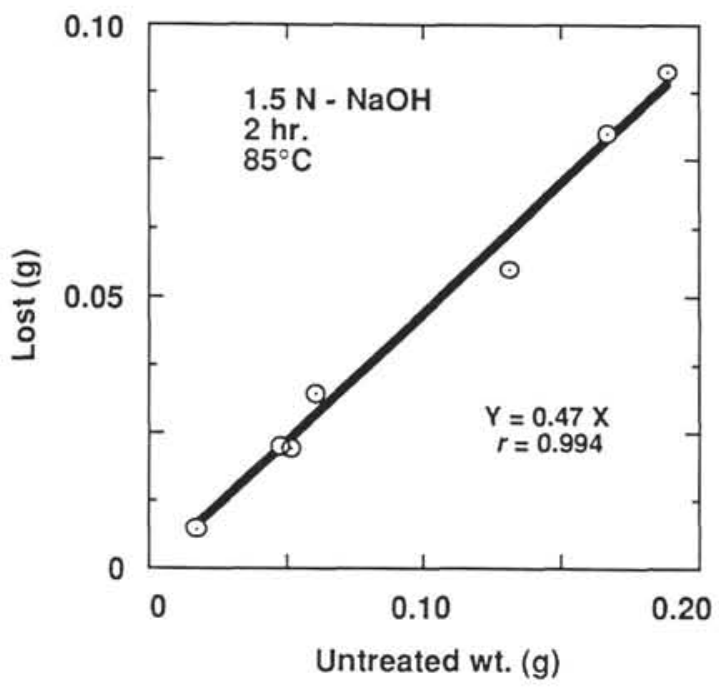

Figure 5. Data used to calculate the mass of crystalline silicate material leached during the procedure used to dissolve biogenic silica from sediments.

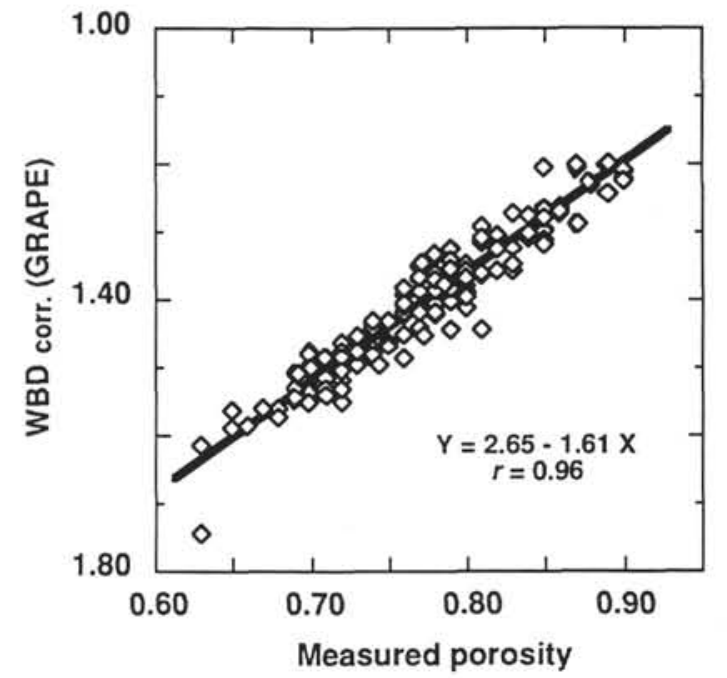

Figure 6. The relationship between porosity measurements determined in our laboratory from volume of water contained in sample to wet-bulk densities calculated from GRAPE measurements (corrected according to Boyce, 1976).

porosity measurements or had desiccated prior to analysis, the relation between gamma ray attenuation porosity evaluator (GRAPE) bulk density (Boyce, 1976) and measured porosity values was used to estimate sample DBD $(R=0.96$, Fig. 6). Linear sedimentation rates were determined using the orbitally tuned time scale provided for each site (Shackleton, this volume). All data used to calculate the flux of eolian minerals is provided in Tables 1 through 4 and Figure 7.

Grain-size distributions were determined for the eolian component using a 256-channel Coulter Counter Multisizer particle-size analyzer and are expressed in phi units $(\phi)$ as mean grain-sizes of a number percent distribution (Tables 1 through 4, Fig. 7). Mean grain-sizes calculated from distributions based on grain volume (not shown) are about $2 \phi$ coarser than number distributions. However, lower precision caused by errors associated with grain volume calculation make number distribution data more preferable. Samples were analyzed in the size range of $9.97 \phi(1 \mu \mathrm{m})$ to $5.06 \phi(30 \mu \mathrm{m})$ and are precise to $\pm 0.04 \phi$, based on replicate analyses.

\section{RESULTS}

The primary objective of Leg 138 was to provide information about responses to global climate changes during the Neogene. To accomplish this, real-time monitoring of the coring process using multiple indicators of lithologic properties ensured complete recovery of the sedimentary section while at sea. In addition, a high-resolution stratigraphic framework was developed for each site which provides the basis for this and many of the paleoclimatic and paleoceanographic studies related to Leg 138 (Mayer, Pisias, Janecek, et al., 1992).

Sediments from the western transect along $110^{\circ} \mathrm{W}$ are composed mostly of diatom- and nannofossil-rich biogenic oozes. Terrigenous mineral content varies with the input of biogenic material and ranges from less than $0.5 \%$ in sediments beneath highly productive areas of the ocean up to $40 \%$ away from these regions. The terrigenous fraction of these sediments comprises a mixture of illite, smectite, plagioclase, and quartz; a more complete description and discussion of the mineralogical changes is provided by Leinen (this volume). Eolian input to these sites provides a continuous history of atmospheric circulation intensity and source region climate throughout the late Neogene and Quaternary. Shipboard description and analysis suggest that hydrothermally related material constitutes a relatively important fraction of the basal sedimentary sequences (Mayer, Pisias, Janecek, et al., 1992). Because minerals associated with this material do not reflect 

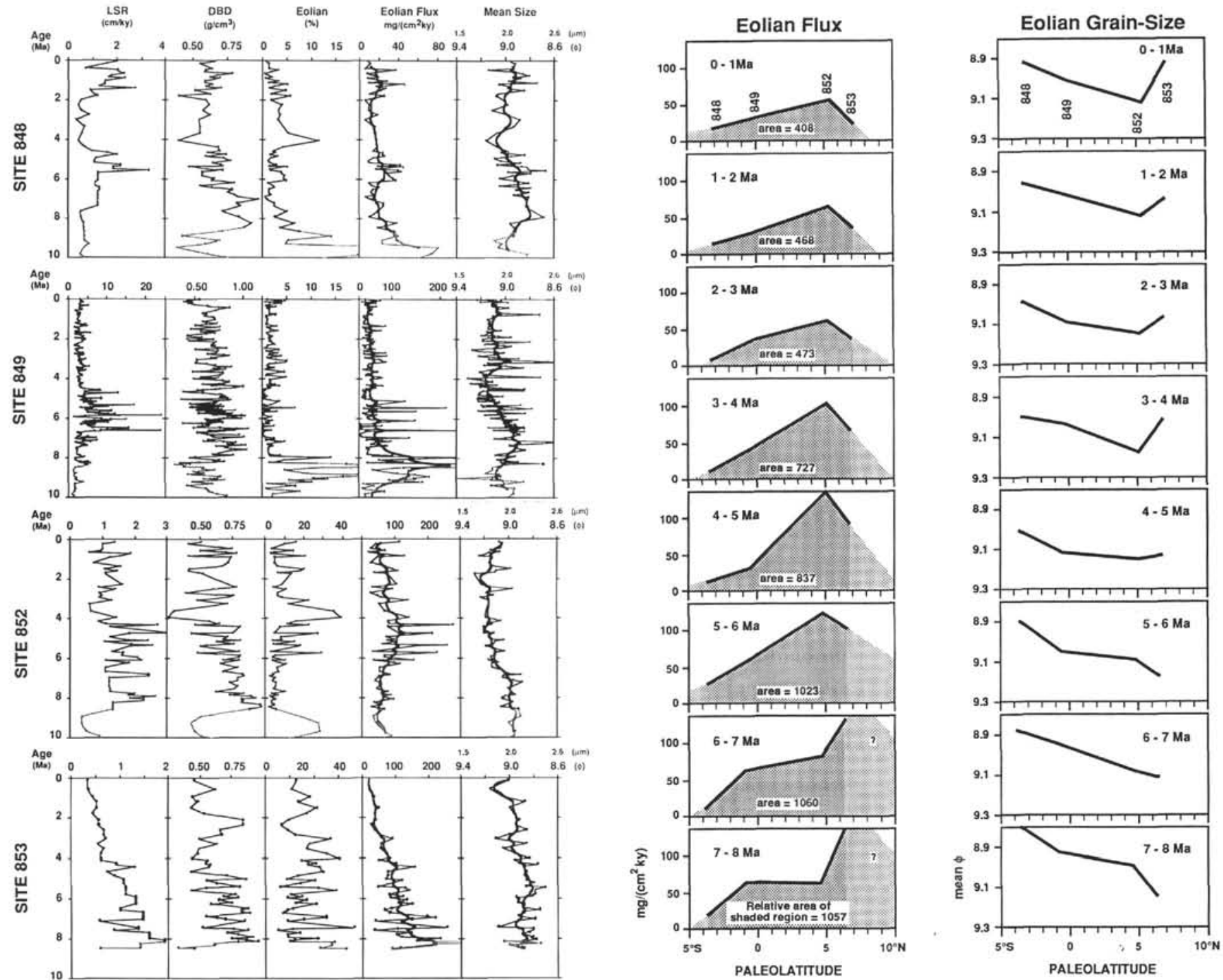

Figure 7. Temporal variations in LSR, DBD, and eolian flux and grain-size in sediments from each site used in this study. Heavy line in flux and grain-size records shows weighted average values for each sample. Lightly shaded line indicates intervals that may contain significant hydrothermal material.

eolian input, accumulation rate and grain-size data are only interpreted in sediments deposited since $8.0 \mathrm{Ma}$.

Eolian accumulation rates range from about 1 to $270 \mathrm{mg}\left(\mathrm{cm}^{2} \cdot \mathrm{k} . \mathrm{y} \text {. }\right)^{-1}$ and show a latitudinal pattern reflecting greater eolian flux near the ITCZ. Average eolian flux for the past 1 m.y. (Fig. 8) is $20 \mathrm{mg}$ $\left(\mathrm{cm}^{2} \cdot \mathrm{k} . \mathrm{y} .\right)^{-1}$ south of the ITCZ at Site 848 , increases to about $55 \mathrm{mg}$ $\left(\mathrm{cm}^{2} \cdot \mathrm{k} . \mathrm{y} .\right)^{-1}$ at Site 852 beneath the ITCZ, and decreases to the north to $25 \mathrm{mg}\left(\mathrm{cm}^{2} \cdot \mathrm{k} . \mathrm{y} \text {. }\right)^{-1}$ at Site 853 . The temporal history of dust flux to each site shows important differences during the late Neogene (Figs. 7 and 8 ). Sediments from Sites 848 and 849 record continuously low dust input throughout most of the interval with accumulation rates generally less than about $50 \mathrm{mg}\left(\mathrm{cm}^{2} \cdot \mathrm{k} . \mathrm{y} .\right)^{-1}$. Eolian accumulation rates in sediments from Site 852 vary from roughly 20 to $100 \mathrm{mg}\left(\mathrm{cm}^{2} \cdot \mathrm{k} . \mathrm{y} \text {. }\right)^{-1}$ for most of the sedimentary section except the part represented by the interval from 3.5 to $6.5 \mathrm{Ma}$, which shows a twofold increase in eolian flux. Eolian accumulation rates from Site 853 average less than $40 \mathrm{mg}$ $\left(\mathrm{cm}^{2} \cdot \mathrm{k} . \mathrm{y} .\right)^{-1}$ during the past $3 \mathrm{~m} . \mathrm{y}$. and gradually increase to more than $125 \mathrm{mg}\left(\mathrm{cm}^{2} \cdot \mathrm{k} . \mathrm{y} .\right)^{-1}$ in the lower section.

The grain-size of eolian material also varies with the modern pattern of zonal wind intensity (Fig. 8, see Fig. 4). Near the ITCZ, atmospheric

Figure 8. Average eolian flux and grain-size data plotted vs. paleolatitude for each 1 m.y. interval through the late Neogene.

winds are less intense and finer eolian material is deposited. Away from the ITCZ, winds are more vigorous and can transport larger eolian particles. Downcore variations in eolian size are strikingly similar between Sites 848 and 849 although eolian material at Site 849 is consistently finer by 0.10 to $0.15 \phi$ (Fig. 7). Each of these records is characterized by relatively constant eolian grain-sizes in the upper portion with a pronounced coarser interval near $3.0 \mathrm{Ma}$. A gradual increase in dust size is recorded in the lower Pliocene, beginning at about 4.0 Ma. Sediments from Site 852 show a similar pattern to those from Sites 848 and 849 although average eolian grain-sizes are finer and less variable than at the southern sites. The record from Site 853, however, shows a different pattern than the other sites, with the coarsest eolian material deposited in the upper portion of the sequence while sediments below are characterized by relatively finer sizes.

\section{DISCUSSION AND PALEOCLIMATIC IMPLICATIONS}

The position of the ITCZ is fundamentally controlled by the balance of trade wind strength in the equatorial region (Fig. 2). On average, the southern trade winds are more intense than their northern counterparts, causing the annual position of the ITCZ to lie north of 
Table 1. Data used to calculate the mass accumulation rate of eolian material and grain-size variations in sediments from Hole 848B.

\begin{tabular}{|c|c|c|c|c|c|c|c|c|c|}
\hline $\begin{array}{l}\text { Core, section. } \\
\text { interval }(\mathrm{cm})\end{array}$ & $\begin{array}{l}\text { Depth } \\
\text { (mbsf) }\end{array}$ & $\begin{array}{l}\text { Depth } \\
\text { (mcd) }\end{array}$ & $\begin{array}{l}\text { Age } \\
(\mathrm{Mi})\end{array}$ & $\begin{array}{c}\mathrm{LSR}^{\mathrm{a}} \\
(\mathrm{cm} / \mathrm{k} . \mathrm{y} .)\end{array}$ & $\begin{array}{c}\mathrm{DBD}^{\mathrm{b}} \\
\left(\mathrm{g} / \mathrm{cm}^{3}\right)\end{array}$ & $\begin{array}{l}\text { Eolian } \\
\text { (\%) }\end{array}$ & $\begin{array}{l}\text { Eol. flux } \\
\left(\mathrm{mg} / \mathrm{cm}^{2} \cdot \mathrm{k} \cdot \mathrm{y} .\right)\end{array}$ & $\begin{array}{c}\text { Mean size } \\
(\mu \mathrm{m})\end{array}$ & $\begin{array}{l}\text { Mean size } \\
\text { (ø) }\end{array}$ \\
\hline \multicolumn{10}{|l|}{ 138-848B- } \\
\hline IH-I. 60-65 & 0.60 & 0.80 & 0.045 & 2.00 & 0.65 & 0.68 & 8.84 & 2.02 & 8.95 \\
\hline $\mathrm{IH}-2.45-49$ & 1.95 & 2.15 & 0.152 & 1.59 & 0.55 & 1.24 & 10.84 & 2.07 & 8.92 \\
\hline $2 \mathrm{H}-1,60-65$ & 2.80 & 5.60 & 0.399 & 0.71 & 0.63 & 1.30 & 5.81 & 2.05 & 8.93 \\
\hline $2 \mathrm{H}-2,60-65$ & 4.30 & 7.10 & 0.474 & 2.06 & 0.59 & 3.10 & 37.68 & 1.78 & 9.14 \\
\hline $2 \mathrm{H}-3,60-65$ & 5.80 & 8.60 & 0.561 & 2.08 & 0.58 & 1.76 & 21.23 & 2.08 & 8.91 \\
\hline $2 \mathrm{H}-4,60-65$ & 7.30 & 10.10 & 0.648 & 2.32 & 0.78 & 0.44 & 7.96 & 2.16 & 8.86 \\
\hline $2 \mathrm{H}-5,60-65$ & 8.80 & 11.60 & 0.731 & 1.80 & 0.67 & 1.19 & 14.35 & 2.24 & 8.80 \\
\hline $2 \mathrm{H}-6,60-65$ & 10.30 & 13.10 & 0.821 & 1.68 & 0.62 & 1.83 & 19.06 & 2.04 & 8.94 \\
\hline $2 \mathrm{H}-7.60-65$ & 11.80 & 14.60 & 0.897 & 2.36 & 0.69 & 0.50 & 8.14 & 2.13 & 8.87 \\
\hline $3 \mathrm{H}-1,60-64$ & 12.30 & 16.25 & 1.014 & 1.52 & 0.58 & 3.14 & 27.68 & 1.99 & 8.98 \\
\hline $3 \mathrm{H}-2,70-76$ & 13.90 & 17.85 & 1.093 & 1.48 & 0.58 & 1.09 & 9.36 & 2.33 & 8.74 \\
\hline $3 \mathrm{H}-3,60-65$ & 15.30 & 19.25 & 1.187 & 1.48 & 0.63 & 4.92 & 45.87 & 1.88 & 9.05 \\
\hline $3 \mathrm{H}-4,60-65$ & 16.80 & 20.75 & 1.284 & 1.26 & 0.59 & 2.32 & 17.25 & 2.00 & 8.97 \\
\hline $3 \mathrm{H}-5,57-62$ & 18.27 & 22.22 & 1.392 & 2.76 & 0.70 & 1.38 & 26.66 & 1.98 & 8.98 \\
\hline $3 \mathrm{H}-6,49-54$ & 19.69 & 23.64 & 1.508 & 0.91 & 0.51 & 4.58 & 21.26 & 2.17 & 8.85 \\
\hline $3 \mathrm{H}-7.60-65$ & 21.30 & 25.25 & 1.692 & 1.04 & 0.62 & 1.61 & 10.38 & 1.87 & 9.06 \\
\hline $4 \mathrm{H}-\mathrm{I}, 58-63$ & 21.78 & 26.13 & 1.786 & 1.18 & 0.39 & 5.57 & 25.63 & 1.85 & 9.08 \\
\hline $4 \mathrm{H}-2,58-63$ & 23.28 & 27.63 & 1.982 & 0.53 & 0.59 & 1.67 & 5.22 & 1.94 & 9.01 \\
\hline $4 \mathrm{H}-3,58-63$ & 24.78 & 29.13 & 2.228 & 0.42 & 0.61 & 3.85 & 9.86 & 1.97 & 8.99 \\
\hline $4 \mathrm{H}-4,58-63$ & 26.28 & 30.63 & 2.521 & 0.53 & 0.57 & 3.14 & 9.49 & 1.81 & 9.11 \\
\hline $4 \mathrm{H}-5,58-63$ & 27.78 & 32.13 & 2.740 & 0.87 & 0.62 & 3.50 & 18.88 & 2.00 & 8.97 \\
\hline $4 \mathrm{H}-6,58-63$ & 29.28 & 33.63 & 2.978 & 0.32 & 0.54 & 3.08 & 5.32 & 2.13 & 8.88 \\
\hline $4 \mathrm{H}-7.58-63$ & 30.78 & 35.13 & 3.343 & 0.68 & 0.55 & 4.19 & 15.67 & 2.05 & 8.93 \\
\hline $5 \mathrm{H}-\mathrm{I} .60-65$ & 31.30 & 36.95 & 3.680 & 0.57 & 0.55 & 5.30 & 16.62 & 1.86 & 9.07 \\
\hline $5 \mathrm{H}-2,60-65$ & 32.80 & 38.45 & 4.052 & 0.40 & 0.39 & 11.49 & 17.92 & 1.75 & 9.16 \\
\hline $5 \mathrm{H}-3,60-65$ & 34.30 & 39.95 & 4.378 & 0.66 & 0.68 & 4.11 & 18.45 & 1.88 & 9.06 \\
\hline $5 \mathrm{H}-4,60-65$ & 35.80 & 41.45 & 4.580 & 0.96 & 0.69 & 2.11 & 13.98 & 1.99 & 8.97 \\
\hline $5 \mathrm{H}-5,60-65$ & 37.30 & 42.95 & 4.661 & 1.59 & 0.60 & 2.55 & 24.33 & 1.91 & 9.04 \\
\hline $5 \mathrm{H}-6,60-65$ & 38.80 & 44.45 & 4.753 & 2.00 & 0.74 & 1.60 & 23.68 & 2.18 & 8.84 \\
\hline $5 \mathrm{H}-7,40-45$ & 40.10 & 45.75 & 4.850 & 1.52 & 0.59 & 2.91 & 26.10 & 2.02 & 8.95 \\
\hline $6 \mathrm{H}-1.61-65$ & 40.81 & $46.5 i$ & 4.907 & 1.16 & 0.72 & 1.71 & 14.28 & 1.84 & 9.09 \\
\hline $6 \mathrm{H}-2,65-69$ & 42.35 & 48.05 & 5.042 & 1.08 & 0.77 & 1.12 & 9.31 & 1.91 & 9.03 \\
\hline $6 \mathrm{H}-3,45-49$ & 43.65 & 49.35 & 5.162 & 1.08 & 0.64 & 1.45 & 10.02 & 2.13 & 8.87 \\
\hline $6 \mathrm{H}-4,93-98$ & 45.63 & 51.33 & 5.267 & 2.16 & 0.70 & 2.76 & 41.73 & 1.87 & 9.07 \\
\hline $6 \mathrm{H}-5,93-97$ & 47.13 & 52.83 & 5,336 & 2.16 & 0.71 & 1.24 & 19.02 & 2.15 & 8.86 \\
\hline $6 \mathrm{H}-6,14-18$ & 47.84 & 53.54 & 5.373 & 1.88 & 0.47 & 5.07 & 44.80 & 2.22 & 8.82 \\
\hline $6 \mathrm{H}-7.40-45$ & 49.60 & 55.30 & 5.467 & 1.88 & 0.60 & 1.45 & 16.36 & 2.23 & 8.81 \\
\hline $7 \mathrm{H}-1,55-60$ & 50.25 & 56.95 & 5.543 & 3,33 & 0.71 & 1.67 & 39.48 & 2.45 & 8.67 \\
\hline $7 \mathrm{H}-2,60-65$ & 51.80 & 58.50 & 5.615 & 2.06 & 0.56 & 3.69 & 42.57 & 1.99 & 8.97 \\
\hline $7 \mathrm{H}-3,60-65$ & 53.30 & 60.00 & 5.692 & 1.24 & 0.57 & 3.96 & 27.99 & 2.26 & 8.79 \\
\hline $7 \mathrm{H}-4,60-65$ & 54.80 & 61.50 & 5.813 & 1.24 & 0.56 & 4.41 & 30.62 & 2.04 & 8.94 \\
\hline $7 \mathrm{H}-5,60-65$ & 56.30 & 63.00 & 5.934 & 1.24 & 0.65 & 4.24 & 34.17 & 1.89 & 9,04 \\
\hline $7 \mathrm{H}-6,60-65$ & 57.80 & 64.50 & 6.055 & 1.24 & 0.63 & 4.82 & 37.65 & 2.06 & 8.92 \\
\hline $7 \mathrm{H}-7,40-45$ & 59.10 & 65.80 & 6.166 & 1.13 & 0.80 & 1.15 & 10.40 & 2.23 & 8.81 \\
\hline $8 \mathrm{H}-\mathrm{I}, 73-77$ & 59.93 & 67.38 & 6.306 & 1.13 & 0.55 & 2.11 & 13.11 & 1.90 & 9.04 \\
\hline $8 \mathrm{H}-2,60-64$ & 61.30 & 68.75 & 6.414 & 131 & 0.63 & 2.44 & 20.14 & 2.26 & 8.79 \\
\hline $8 \mathrm{H}-3,56-60$ & 62.76 & 70.21 & 6.526 & 1.31 & 0.78 & 1.37 & 14.00 & 2.08 & 8.91 \\
\hline $8 \mathrm{H}-4,60-64$ & 64.30 & 71.75 & 6.648 & 1.23 & 0.75 & 1.34 & 12.36 & 2.23 & 8.81 \\
\hline $8 \mathrm{H}-5,60-64$ & 65.80 & 73.25 & 6.771 & 1.23 & 0.80 & 1.60 & 15.74 & 2.06 & 8.93 \\
\hline $8 \mathrm{H}-6,66-70$ & 67.36 & 74.81 & 6.898 & 1.23 & 0.82 & 0.60 & 6.05 & 2.23 & 8.81 \\
\hline $8 \mathrm{H}-7,42-46$ & 68.62 & 76.07 & 7.000 & 1.23 & 0.97 & 0.98 & 11.69 & & \\
\hline $9 \mathrm{H}-\mathrm{I}, 60-65$ & 69.30 & 78.90 & 7.231 & 1.23 & 0.82 & 2.44 & 24.61 & & \\
\hline $9 \mathrm{H}-2,60-65$ & 70.80 & 80.40 & 7.353 & 1.23 & 0.84 & 2.38 & 24.59 & 2.20 & 8.83 \\
\hline $9 \mathrm{H}-3,98-103$ & 72.68 & 82.28 & 7.624 & 0.53 & 0.71 & 5.09 & 19.15 & & \\
\hline $9 \mathrm{H}-4,98-103$ & 74.18 & $\begin{array}{l}02.20 \\
83.78\end{array}$ & 7.909 & 0.53 & 0.83 & 2.33 & 10.25 & 2.43 & 8.69 \\
\hline $9 \mathrm{H}-5,60-65$ & 75.30 & 84.90 & 8.206 & 0.63 & 0.92 & 6.52 & 37.79 & 2.01 & 8.96 \\
\hline $9 \mathrm{H}-6,60-65$ & 76.80 & 86.40 & 8.442 & 0.63 & 0.87 & 3.50 & 19.18 & 2.06 & 8.93 \\
\hline $9 \mathrm{H}-7,54-59$ & 78.24 & 87.84 & 8.667 & 0.67 & 0.71 & 7.54 & 35.87 & 2.07 & 8.91 \\
\hline $10 \mathrm{H}-1.57-62$ & 78.77 & 89.37 & 8.896 & 0.67 & 0.42 & 14.09 & 39.65 & 2.05 & 8.93 \\
\hline $10 \mathrm{H}-2.56-61$ & 80.26 & 90.86 & 9.121 & 0.66 & 0.69 & 5.18 & 23.59 & 1.83 & 9.09 \\
\hline $\mathrm{IOH}-3,90-95$ & 82.10 & 92.70 & 9.306 & 0.85 & 0.59 & 4.73 & 23.72 & 1.92 & 9.02 \\
\hline $10 \mathrm{H}-4,96-101$ & 83.66 & 94.26 & 9.491 & 0.66 & 0.38 & 32.45 & 81.38 & 1.87 & 9.06 \\
\hline $10 \mathrm{H}-5,58-63$ & 84.78 & 95.38 & 9.811 & 0.50 & 0.60 & 24.43 & 73.29 & 2.22 & 8.82 \\
\hline $\mathrm{IOH}-6,58-63$ & 86.28 & 96.88 & 10.020 & 0.72 & 0.78 & 10.44 & 58.63 & 2.19 & 8.83 \\
\hline $10 \mathrm{H}-7.52-57$ & 87.72 & 98.32 & 10.219 & 0.72 & 0.75 & 21.82 & 117.83 & 1.87 & 9.06 \\
\hline $11 \mathrm{H}-1.94-99$ & 88.64 & 100.99 & 10.589 & 0.72 & 0.81 & $\begin{array}{r}21.02 \\
7.71\end{array}$ & 44.96 & 1.84 & 9.09 \\
\hline $11 \mathrm{H}-2.59-64$ & 89.79 & 102.14 & 10.748 & 0.72 & 0.79 & 12.25 & 69.68 & 1.73 & 9.18 \\
\hline IIH-3, 59-64 & 91.29 & 103.64 & 10.989 & 0.63 & 089 & 11.50 & 64.48 & 1.86 & 9.07 \\
\hline $1 \mathrm{H}-4,1-6$ & 92.21 & 104.56 & 11.121 & 0.70 & 0.92 & 6.90 & 44.44 & 1.86 & 9.07 \\
\hline $11 \mathrm{H}-5.6-11$ & 92.79 & 105.14 & 11.204 & 0.70 & 0.98 & 6.58 & 45.14 & 1.71 & 9.19 \\
\hline
\end{tabular}

"LSR = linear sedimentation rate,

${ }^{\mathrm{b}} \mathrm{DBD}=$ dry-bulk density.

the equator. The underlying cause of the hemispheric circulation asymmetry is the different temperature gradients between each of the poles and the equator. Significant differences in heat and radiation budgets cause the annual mid-tropospheric temperatures above Antarctica to be more than $11^{\circ} \mathrm{C}$ colder than above the central Arctic Ocean (Flohn, 1981). With the possible exception of the glacial/inter- glacial oscillation of the late Quaternary, these differences have been equal or greater throughout the late Neogene. Thus, an important question remains: How far north was the ITCZ in the geologic past?

Because latitudinal patterns of eolian material in the equatorial Pacific closely follow the position of the ITCZ, the spatial pattern of eolian deposition may prove useful in tracking the position of the 
Table 2. Data used to calculate the mass accumulation rate of eolian material and eolian grain-size variations in sediments from Hole 849B.

\begin{tabular}{|c|c|c|c|c|c|c|c|c|c|}
\hline $\begin{array}{l}\text { Core, section, } \\
\text { interval }(\mathrm{cm})\end{array}$ & $\begin{array}{l}\text { Depth } \\
\text { (mbsf) }\end{array}$ & $\begin{array}{l}\text { Depth } \\
\text { (med) }\end{array}$ & $\begin{array}{l}\text { Age } \\
\text { (Ma) }\end{array}$ & $\begin{array}{l}\mathrm{LSR}^{\mathrm{i}} \\
(\mathrm{cm} / \mathrm{k} . \mathrm{y} .)\end{array}$ & $\begin{array}{c}\mathrm{DBD}^{\mathrm{b}} \\
\left(\mathrm{g} / \mathrm{cm}^{3}\right)\end{array}$ & $\begin{array}{l}\text { Eolian } \\
\text { (\%) }\end{array}$ & $\begin{array}{c}\text { Eol. flux } \\
\left(\mathrm{mg} / \mathrm{cm}^{2} \cdot \mathrm{k}_{\left.\text {. } \mathrm{y}_{\text {. }}\right)}\right.\end{array}$ & $\begin{array}{l}\text { Mean size } \\
(\mu \mathrm{m})\end{array}$ & $\begin{array}{c}\text { Mean size } \\
\text { (ф) }\end{array}$ \\
\hline 138-849B- & & & & & & & & & \\
\hline IH-1.60-65 & 0.60 & 0.60 & 0.020 & 2.92 & 0.55 & 0.97 & 15.56 & 1.67 & 9.23 \\
\hline IH-2, 60-65 & 2.10 & 2.10 & 0.068 & 3.27 & 0.42 & 4.64 & 63.63 & 1.91 & 9.03 \\
\hline IH-3.60-65 & 3.60 & 3.60 & 0.116 & 3.82 & 0.39 & 0.51 & 7.56 & 1.69 & 9.21 \\
\hline IH-4,60-65 & 5.10 & 5.10 & 0.158 & 3.65 & 0.48 & 1.60 & 28.00 & 1.98 & 8.98 \\
\hline IH-5, 60-65 & 6.60 & 6.60 & 0.200 & 3.06 & 0.43 & 3.87 & 50.81 & 1.82 & 9.10 \\
\hline $2 \mathrm{H}-1.49-53$ & 7.19 & 9.14 & 0.283 & 2.83 & 0.50 & 1.21 & 17.21 & 1.86 & 9.07 \\
\hline $2 \mathrm{H}-2,69-73$ & 8.89 & 10.84 & 0.340 & 5.22 & 0.63 & 0.74 & 24.42 & 1.81 & 9.11 \\
\hline $2 \mathrm{H}-3.60-64$ & 10.30 & 12.25 & 0.381 & 2.97 & 0.65 & 2.78 & 53.82 & & \\
\hline $2 \mathrm{H}-4.64-68$ & 11.84 & 13.79 & 0.442 & 2.83 & 0.86 & 1.02 & 24.91 & 1.99 & 8.97 \\
\hline $2 \mathrm{H}-5,60-64$ & 13.30 & 15.25 & 0.491 & 3.53 & 0.75 & 1.49 & 39.47 & 1.86 & 9.07 \\
\hline $3 \mathrm{H}-1,102-106$ & 17.22 & 21.27 & 0.715 & 3.00 & 0.58 & 1.53 & 26.64 & 1.90 & 9.04 \\
\hline $3 \mathrm{H}-2,66-70$ & 18.36 & 22.41 & 0.758 & 2.35 & 0.62 & 2.70 & 39.32 & 2.37 & 8.72 \\
\hline $3 \mathrm{H}-3,60-64$ & 19.80 & 23.85 & 0.827 & 2.75 & 0.60 & 0.78 & 12.93 & 1.82 & 9.10 \\
\hline $3 \mathrm{H}-5,60-64$ & 22.80 & 26.85 & 0.936 & 2.50 & 0.70 & 0.88 & 15.35 & 1.83 & 9.10 \\
\hline $3 \mathrm{H}-6.57-61$ & 24.27 & 28.32 & 1.002 & 2.31 & 0.71 & 1.11 & 18.31 & 1.77 & 9.15 \\
\hline $4 \mathrm{H}-1,58-62$ & 26.28 & 31.33 & 1.106 & 2.91 & 0.58 & 0.92 & 15.51 & 1.79 & 9.13 \\
\hline $4 \mathrm{H}-2,113-117$ & 28.33 & 33.38 & 1.196 & 2.00 & 0.73 & 0.97 & 14.16 & 1.78 & 9.13 \\
\hline $4 \mathrm{H}-3,124-128$ & 29.94 & 34.99 & 1.253 & 3.25 & 0.67 & 0.75 & 16.42 & 1.80 & 9.12 \\
\hline $4 \mathrm{H}-4,18-22$ & 30.38 & 35.43 & 1.266 & 4.40 & 0.62 & 0.89 & 24.32 & 1.78 & 9.13 \\
\hline $4 \mathrm{H}-6,59-63$ & 33.79 & 38.84 & 1.355 & 4.88 & 0.57 & 2.92 & 81.23 & 1.79 & 9.12 \\
\hline $4 \mathrm{H}-7,17-21$ & 34.87 & 39.92 & 1.390 & 1.64 & 0.76 & 0.86 & 10.64 & 1.77 & 9.14 \\
\hline $5 \mathrm{H}-1,60-64$ & 35.80 & 42.25 & 1.511 & 2.69 & 0.46 & 1.67 & 20.60 & 1.77 & 9.14 \\
\hline $5 \mathrm{H}-2.60-64$ & 37.30 & 43.75 & 1.565 & 2.82 & 0.76 & 3.15 & 67.44 & 1.91 & 9.03 \\
\hline $5 \mathrm{H}-3,60-64$ & 38.80 & 45.25 & 1.626 & 1.90 & 0.56 & 1.72 & 18.23 & 1.76 & 9.15 \\
\hline $5 \mathrm{H}-4,60-64$ & 40.30 & 46.75 & 1.698 & 5.71 & 0.71 & 0.95 & 38.58 & 1.80 & 9.12 \\
\hline $5 \mathrm{H}-6,66-70$ & 43.36 & 49.81 & 1.800 & 3.24 & 0.43 & 1.48 & 20.65 & 1.70 & 9.20 \\
\hline $5 \mathrm{H}-7,60-64$ & 44.80 & 51.25 & 1.847 & 4.00 & 0.42 & 2.11 & 35.43 & 1.88 & 9.06 \\
\hline $6 \mathrm{H}-1.94-99$ & 45.64 & 53.14 & 1.896 & 3.84 & 0.57 & 1.58 & 34.59 & 2.01 & 8.96 \\
\hline $6 \mathrm{H}-2.60-65$ & 46.80 & 54.30 & 1.937 & 1.81 & 0.55 & 1.29 & 12.82 & 1.77 & 9.14 \\
\hline $6 \mathrm{H}-4,116-121$ & 50.36 & 57.86 & 2.021 & 3.60 & 0.69 & 1.70 & 42.25 & 1.72 & 9.18 \\
\hline $6 \mathrm{H}-6.60-65$ & 52.80 & 60.30 & 2.081 & 3.64 & 0.44 & 1.54 & 24.62 & 1.72 & 9.19 \\
\hline $6 \mathrm{H}-7,60-65$ & 54.30 & 61.80 & 2.139 & 3.64 & 0.72 & 1.84 & 48.29 & 2.25 & 8.79 \\
\hline $7 \mathrm{H}-\mathrm{I} .60-65$ & 54.80 & 64.25 & 2,258 & 2.31 & 0.68 & 2.60 & 40.86 & 1.74 & 9.17 \\
\hline $7 \mathrm{H}-2,60-65$ & 56,30 & 65.75 & 2.315 & 1.70 & 0.71 & 1.04 & 12.60 & 1.94 & 9.01 \\
\hline $7 \mathrm{H}-3,60-65$ & 57.80 & 67.25 & 2.380 & 2.76 & 0.68 & 1.07 & 20.18 & 2.02 & 8.95 \\
\hline $7 \mathrm{H}-4,60-65$ & 59.30 & 68.75 & 2.437 & 3.65 & 0.83 & 0.51 & 15.55 & 1.87 & 9.06 \\
\hline $7 \mathrm{H}-5,60-65$ & 0.80 & 70.25 & 2.486 & 2.41 & 0.73 & 2.75 & 48.35 & 1.71 & 9.19 \\
\hline $7 \mathrm{H}-6,60-65$ & 62.30 & 71.75 & 2.536 & 2.00 & 0.51 & 3.36 & 34.31 & 1.62 & 9.27 \\
\hline $7 \mathrm{H}-7,60-65$ & 63.80 & 73.25 & 2.602 & 2.09 & 0.74 & 0.83 & 12.87 & 1.68 & 9.21 \\
\hline $8 \mathrm{H}-\mathrm{I}, 56-60$ & 64.26 & 74.96 & 2.684 & 3.58 & 0.72 & 3.19 & 82.22 & 1.93 & 9.01 \\
\hline $8 \mathrm{H}-2,56-60$ & 65.76 & 76.46 & 2.730 & 1.67 & 0.63 & 1.40 & 14.67 & 1.83 & 9.09 \\
\hline $8 \mathrm{H}-3,56-60$ & 67.26 & 77.96 & 2.775 & 3.91 & 0.80 & 1.38 & 43.11 & 1.84 & 9.09 \\
\hline $8 \mathrm{H}-4,56-60$ & 68.76 & 79.46 & 2.848 & 2.60 & 0,85 & 3.83 & 84.74 & 1.67 & 9.22 \\
\hline $8 \mathrm{H}-5,56-60$ & 70.26 & 80.96 & 2.911 & 2,73 & 0.67 & 0.88 & 16.03 & 1.83 & 9.10 \\
\hline $8 \mathrm{H}-6,56-60$ & 71.76 & 82.46 & 2.968 & 2.70 & 0.72 & 1.62 & 31.56 & 2.10 & 8.89 \\
\hline $8 \mathrm{H}-7.56-60$ & 3.26 & 83.96 & 3.025 & 4.36 & 0.52 & 2.75 & 62.40 & 1.77 & 9.14 \\
\hline $9 \mathrm{H}-1.58-63$ & 73.78 & 85.43 & 3.079 & 2.55 & 0.53 & 4,97 & 67.10 & & \\
\hline $9 \mathrm{H}-2.58-63$ & 75.28 & 86.93 & 3.140 & 3.14 & 0.52 & 4.91 & 80.25 & 3.05 & 8.36 \\
\hline $9 \mathrm{H}-3,58-63$ & 76.78 & 88.43 & 3.191 & 3.33 & 0.51 & 2.76 & 46.94 & 1.76 & 9.15 \\
\hline $9 \mathrm{H}-4,58-63$ & 78.28 & 89.93 & 3.238 & 3.15 & 0.62 & 1.53 & 29.86 & 1.92 & 9.02 \\
\hline $9 \mathrm{H}-5,58-63$ & 79.78 & 91.43 & 3.290 & 3.60 & 0.71 & 1.12 & 28.59 & 1.73 & 9.17 \\
\hline $9 \mathrm{H}-6,58-63$ & 81.28 & 92.93 & 3.348 & 3.82 & 0.70 & 0.91 & 24.20 & 1.96 & 9.00 \\
\hline $10 \mathrm{H}-\mathrm{I} .59-64$ & 83.29 & 95.09 & 3.426 & 2.19 & 0.74 & 2.44 & 39.62 & 2.15 & 8.86 \\
\hline $10 \mathrm{H}-2,59-64$ & 84.79 & 96.59 & 3.482 & 3.69 & 0.69 & 0.99 & 25.11 & & \\
\hline $10 \mathrm{H}-3,59-64$ & 86.29 & 98.09 & 3.539 & 2.40 & 0.82 & 0.59 & 11.60 & 1.82 & 9.10 \\
\hline $10 \mathrm{H}-5,59-64$ & 89.29 & 101.09 & 3.645 & 2.57 & 0.48 & 4.43 & 54.66 & 1.68 & 9.21 \\
\hline $10 \mathrm{H}-7,45-50$ & 92.15 & 103,95 & 3.752 & 3.00 & 0.79 & 1.04 & 24.57 & 1.74 & 9.16 \\
\hline $11 \mathrm{H}-16,0-65$ & 92.80 & 106.40 & 3.829 & 3.43 & 0.76 & 0.69 & 18.09 & 1.72 & 9.18 \\
\hline $11 \mathrm{H}-2,60-65$ & 94.30 & 107.90 & 3.884 & 2.28 & 0.56 & 4.77 & 60.78 & 2.38 & 8.71 \\
\hline $11 \mathrm{H}-3,60-65$ & 95.80 & 109.40 & 3.941 & 2.48 & 0.74 & 3.75 & 68.73 & 1.58 & 9.31 \\
\hline $11 \mathrm{H}-5,60-65$ & 98.80 & 112.40 & 4.053 & 3.05 & 0.69 & 1.54 & 32.31 & 1.69 & 9.20 \\
\hline $11 \mathrm{H}-7,53-58$ & 101.73 & 115.33 & 4.158 & 2.76 & 0,85 & 1.73 & 40.63 & 1.81 & 9.11 \\
\hline $12 \mathrm{H}-1,60-65$ & 102.30 & 116.75 & 4.208 & 3.64 & 0.81 & 1.45 & 42.69 & 1.60 & 9.29 \\
\hline $12 \mathrm{H}-2.601-65$ & 103.80 & 118.25 & 4.259 & 3.31 & 0.58 & 2.62 & 50.20 & 1.75 & 9.16 \\
\hline $12 \mathrm{H}-3,60-65$ & 105.30 & 119.75 & 4,309 & 2.78 & 0.80 & 0.97 & 21.68 & 1.86 & 9.07 \\
\hline $12 \mathrm{H}-4,60-65$ & 106.80 & 121.25 & 4.346 & 3.01 & 0.68 & 3.52 & 72.13 & 1.68 & 9.21 \\
\hline $12 \mathrm{H}-5,59-64$ & 108.29 & 122.74 & 4.395 & 3.01 & 1.00 & 1.12 & 33.66 & 1.69 & 9.21 \\
\hline $12 \mathrm{H}-6,59-64$ & 109.79 & 124.24 & 4.457 & 3.50 & 0.90 & 1.63 & 51.33 & 1.90 & 9.04 \\
\hline $12 \mathrm{H}-7,60-65$ & 111.30 & 125.75 & 4.499 & 4.09 & 0.71 & 0.91 & 26.42 & 2.18 & 8.84 \\
\hline $13 \mathrm{H}-1,57-62$ & 111.77 & 126.22 & 4.511 & 4.09 & 0.76 & 0.58 & 18.13 & 1.77 & 9.14 \\
\hline $13 \mathrm{H}-2,58-63$ & 113.28 & 127.73 & 4.538 & 8.00 & 0.53 & 0.56 & 23.74 & & \\
\hline $13 \mathrm{H}-3,58-63$ & 114.78 & 129.23 & 4.557 & 8.00 & 0.58 & 0.23 & 10.47 & 1.82 & 9.10 \\
\hline $13 \mathrm{H}-4,58-63$ & 116.28 & 130.73 & 4.575 & 8.48 & 0.56 & 0.57 & 27.25 & 2.17 & 8.84 \\
\hline $13 \mathrm{H}-5,57-62$ & 117.77 & 132.22 & 4.593 & 8.48 & 0.59 & 0.40 & 19.79 & 2.16 & 8.86 \\
\hline $13 \mathrm{H}-6,57-62$ & 119.27 & 133.72 & 4.616 & 6.09 & 0.59 & 0.30 & 10.61 & 1.72 & 9.19 \\
\hline $14 X-3,61-66$ & 124.31 & 138.76 & 4.708 & 12.92 & 0.58 & 0.27 & 20.47 & 1.68 & 9.21 \\
\hline $15 X-1,60-64$ & 130.90 & 145.35 & 4.776 & 3.89 & 0.77 & 0.47 & 14.12 & 1.67 & 9.22 \\
\hline $15 X-2,60-64$ & 132.40 & 146.85 & 4.819 & 4.44 & 0.79 & 0.35 & 12.31 & 1.74 & 9.16 \\
\hline $15 X-3,60-64$ & 133.90 & 148.35 & 4.871 & 2.67 & 0.82 & 0.90 & 19.75 & 1.92 & 9.02 \\
\hline $15 X-4,60-64$ & 135.40 & 149.85 & 4.917 & 3.91 & 0.76 & 0.26 & 7.60 & 1.68 & 9.22 \\
\hline $15 X-5,60-64$ & 136.90 & 151.35 & 4.961 & 4.32 & 0.82 & 0.50 & 17.65 & 1.79 & 9.13 \\
\hline $15 \times-6,60-64$ & 138.40 & 152.85 & 4.988 & 8.52 & 0.84 & 0.24 & 16.97 & 1.66 & 9.23 \\
\hline $16 \times-3,60-64$ & 143.60 & 158.05 & 5.085 & 3.33 & 0.87 & 1.65 & 47.73 & 1.83 & 9.09 \\
\hline $16 \times-4,35-39$ & 144.85 & 159.30 & 5.113 & 5.77 & 0.70 & 3.48 & 140.42 & 1.67 & 9.22 \\
\hline $17 X-3.54-59$ & 153.24 & 167.69 & 5.286 & 5.28 & 0.52 & 1.71 & 46.90 & 1.84 & 9.08 \\
\hline $17 \times-4,60-65$ & 154.80 & 169.25 & 5.305 & 17.00 & 0.68 & 0.39 & 45.07 & 1.70 & 9.20 \\
\hline
\end{tabular}


Table 2 (continued).

\begin{tabular}{|c|c|c|c|c|c|c|c|c|c|}
\hline $\begin{array}{l}\text { Core, section. } \\
\text { interval (cm) }\end{array}$ & $\begin{array}{l}\text { Depth } \\
\text { (mbsf) }\end{array}$ & $\begin{array}{l}\text { Depth } \\
\text { (mcd) }\end{array}$ & $\begin{array}{l}\text { Age } \\
(\mathrm{Mia})\end{array}$ & $\begin{array}{c}\mathrm{LSR}^{\mathrm{al}} \\
(\mathrm{cm} / \mathrm{k} . \mathrm{y} .)\end{array}$ & $\begin{array}{l}\mathrm{DBD}^{\mathrm{b}} \\
\left(\mathrm{g} / \mathrm{cm}^{3}\right)\end{array}$ & $\begin{array}{c}\text { Eolian } \\
(\%)\end{array}$ & $\begin{array}{c}\text { Eol. flux } \\
\left(\mathrm{mg} / \mathrm{cm}^{2} \cdot \mathrm{k} . \mathrm{y} .\right)\end{array}$ & $\begin{array}{c}\text { Mean size } \\
(\mu \mathrm{m})\end{array}$ & $\begin{array}{c}\text { Mean size } \\
(0)\end{array}$ \\
\hline $17 X-5,90-95$ & 156.60 & 171.05 & 5.315 & 17.00 & 0.77 & 0.17 & 22.11 & 2.14 & 8.87 \\
\hline $17 \times-6.70-75$ & 157.90 & 172.35 & 5.325 & 9.77 & 0.63 & 0.84 & 51.62 & 1.88 & 9.06 \\
\hline $18 X-3.103-107$ & 163.33 & 177.78 & 5.398 & 10.23 & 0.80 & 0.78 & 63.54 & 1.95 & 9.00 \\
\hline $18 X-4,61-65$ & 64.41 & 178.86 & 5.408 & 10.23 & 0.47 & 0.54 & 26.02 & & \\
\hline $18 X-6.95-99$ & 167.75 & 182.20 & 5.470 & 6.36 & 0.74 & 0.27 & 12.92 & 2.27 & 8.78 \\
\hline $19 \times-2,95-101$ & 70.95 & 185.40 & 5.542 & 4.52 & 0.78 & 1.47 & 51.74 & 1.95 & 9.00 \\
\hline $19 \times-4,14-19$ & 173.14 & 187.59 & 5.575 & 7.24 & 0.92 & 0.47 & 31.08 & 1.67 & 9.22 \\
\hline $19 X-5,90-95$ & 175.40 & 189.85 & 5.599 & 11.25 & 0.80 & 0.56 & 50.51 & & \\
\hline $20 X-1.59-64$ & 178.29 & 192.74 & 5.640 & 8.16 & 0.71 & 3.01 & 174.32 & 1.83 & 9.09 \\
\hline $20 \times-2.60-65$ & 179.80 & 194.25 & 5.665 & 8.16 & 0.70 & 0.48 & 27.26 & 1.91 & 9.03 \\
\hline $20 \mathrm{X}-4,18-23$ & 182.38 & 196.83 & 5.708 & 5.00 & 0.89 & 0.23 & 10.23 & 2.19 & 8.84 \\
\hline $21 X-1,82-87$ & 188.12 & 202.57 & 5.779 & 12.05 & 0.87 & 0.19 & 20.07 & 1.87 & 9.07 \\
\hline $21 X-2,82-87$ & 189.62 & 204.07 & 5.791 & 12.05 & 0.75 & 0.45 & 40.31 & 1.74 & 9.17 \\
\hline IX-3,59-64 & 190.89 & 205.34 & 5.798 & 24.05 & 0.69 & 0.32 & 53.54 & 1.92 & 9.02 \\
\hline $22 \times-4,60-65$ & 202.10 & 216.55 & 5.916 & 1.50 & 0.89 & 1.86 & 24.87 & 1.95 & 9.00 \\
\hline $22 X-5.90-95$ & 203.90 & 218.35 & 5.984 & 8.63 & 0.98 & 0.32 & 26.69 & 1.80 & 9.12 \\
\hline $23 \times-1,60-65$ & 207.20 & 221.65 & 6.023 & 8.63 & 0.63 & 0.63 & 34.34 & 2.10 & 8.90 \\
\hline $23 X-5,60-65$ & 213.20 & 227.65 & 6.075 & 11.00 & 0.77 & 2.02 & 171.07 & 1.93 & 9.02 \\
\hline $24 X-2,60-65$ & 218.40 & 232.85 & 6.157 & 8.75 & 0.78 & 1.33 & 90.68 & 2.03 & 8.95 \\
\hline $24 X-4,60-65$ & 221.40 & 235.85 & 6.202 & 10.25 & 0.79 & 0.76 & 61.14 & 1.94 & 9.01 \\
\hline $24 X-7,39-44$ & 225.69 & 240.14 & 6.269 & 4.09 & 0.67 & 1.52 & 41.63 & 1.95 & 9.00 \\
\hline $25 X-1.52-57$ & 226.52 & 240.97 & 6.289 & 3.25 & 0.62 & 0.14 & 2.81 & 2.07 & 8.92 \\
\hline $25 X-3,60-65$ & 229.60 & 244.05 & 6.353 & 5.00 & 0.65 & 0.16 & 5.29 & 1.96 & 9.00 \\
\hline $25 X-4,60-65$ & 231.10 & 245.55 & 6.383 & 5.00 & 0.58 & 2.42 & 70.30 & 2.08 & 8.91 \\
\hline $25 x-5,60-65$ & 232.60 & 247.05 & 6.419 & 5.53 & 0.54 & 0.11 & 3.17 & 2.17 & 8.84 \\
\hline $25 X-6,60-65$ & 234.10 & 248.55 & 6.444 & 6.67 & 0.59 & 0.22 & 8.68 & 1.77 & 9.14 \\
\hline $26 \mathrm{X}-1.58-63$ & 236.28 & 250.73 & 6.477 & 6.32 & 0.49 & 0.26 & 8.05 & 2.18 & 8.84 \\
\hline $27 X-1,59-64$ & 245.89 & 260.34 & 6.545 & 11.58 & 0.94 & 0.04 & 3.97 & 2.11 & 8.89 \\
\hline $27 X-5,59-64$ & 251.89 & 266.34 & 6.599 & 23.80 & 0.80 & 1.85 & 351.86 & 1.83 & 9.10 \\
\hline $28 X-1,60-65$ & 255.60 & 270.05 & 6.615 & 23.80 & 0.66 & 1.18 & 185.60 & 2.03 & 8.95 \\
\hline $28 \mathrm{X}-3,18-23$ & 258.18 & 272.63 & 6.634 & 8.46 & 0.64 & 0.34 & 18.21 & 2.18 & 8.84 \\
\hline $28 X-4,24-29$ & 259.74 & 274.19 & 6.653 & 6.82 & 0.82 & 0.46 & 25.82 & 2.04 & 8.94 \\
\hline $28 \times-6,135-140$ & 263.85 & 278.30 & 6.805 & 3.25 & 0.63 & 0.01 & 0.13 & 2.12 & 8.88 \\
\hline $29 X-2,58-63$ & 266.78 & 281.23 & 6.900 & 2.34 & 0.69 & 0.52 & 8.44 & 2.03 & 8.95 \\
\hline $29 X-4,62-67$ & 269.82 & 284.27 & 6.971 & 7.27 & 0.86 & 2.46 & 153.88 & 1.96 & 9.00 \\
\hline $29 \times-5,62-67$ & 271.32 & 285.77 & 7.023 & 2.00 & 0.80 & 2.66 & 42.50 & 1.84 & 9.09 \\
\hline $29 \times-6,58-63$ & 272.78 & 287.23 & 7.083 & 4.15 & 0.64 & 0.83 & 22.16 & 2.07 & 8.92 \\
\hline $29 X-7,18-23$ & 273.88 & 288.33 & 7.110 & 4.15 & 0.70 & 2.38 & 69.14 & 2.06 & 8.92 \\
\hline $30 X-2,84-89$ & 276.64 & 291.09 & 7.159 & 3.64 & 0.59 & 4.61 & 98.84 & 2.94 & 8.41 \\
\hline $30 \times-4.64-69$ & 279.44 & 293.89 & 7.217 & 1.97 & 0.93 & 2.74 & 50.27 & & \\
\hline $30 X-5,58-63$ & 280.88 & 295.33 & 7.285 & 3.86 & 0.84 & 1.13 & 36.62 & 2.13 & 8.87 \\
\hline $30 \times-7,17-22$ & 283.47 & 297.92 & 7.347 & 4.40 & 1.03 & 1.78 & 80.81 & 2.01 & 8.96 \\
\hline $31 \times-1.60-65$ & 284.50 & 298.95 & 7.369 & 4.89 & 0.85 & 2.10 & 87.35 & 2.00 & 8.96 \\
\hline $31 X-2,90-95$ & 286.30 & 300.75 & 7.407 & 3.56 & 0.72 & 2.27 & 58.16 & 2.09 & 8.90 \\
\hline $31 X-3,90-95$ & 287.80 & 302.25 & 7.449 & 3.00 & 0.84 & 1.42 & 35.73 & & \\
\hline $31 \times-4,45-50$ & 288.85 & 303.30 & 7.489 & 2.05 & 0.82 & 1.34 & 22.49 & 2.09 & 8.90 \\
\hline $31 X-5,59-64$ & 290.49 & 304.94 & 7.562 & 2.28 & 1.04 & 1.32 & 31.31 & 2.20 & 8.83 \\
\hline $31 X-C C, 2-7$ & 293.44 & 307.89 & 7.807 & 2.39 & 0.69 & 1.51 & 24.83 & 1.88 & 9.05 \\
\hline $32 X-1,91-95$ & 294.51 & 308.96 & 7.847 & 3.12 & 0.90 & 3.64 & 102.32 & 1.85 & 9.08 \\
\hline $32 X-2,90-94$ & 296.00 & 310.45 & 7.894 & 3.20 & 0.75 & 1.76 & 42.21 & 2.07 & 8.92 \\
\hline $32 \times-5,90-94$ & 300.50 & 314.95 & 8.079 & 1.83 & 0.76 & 4.40 & 61.27 & 1.87 & 9.06 \\
\hline $32 \times-7,29-33$ & 302.89 & 317.34 & 8.191 & 5.00 & 0.81 & 3.34 & 135.20 & 2.11 & 8.89 \\
\hline $33 \mathrm{X}-1,90-94$ & 304.10 & 318.55 & 8.220 & 2.96 & 0.55 & 1.50 & 24.47 & 1.90 & 9.04 \\
\hline $33 \mathrm{X}-3,98-102$ & 307.18 & 321.63 & 8.296 & 4.32 & 0.45 & 17.41 & 338.40 & 2.43 & 8.69 \\
\hline $33 \times-4,91-95$ & 308.61 & 323.06 & 8.327 & 5.17 & 0.29 & 22.08 & 331.16 & 2.00 & 8.97 \\
\hline $33 X-7,32-36$ & 312.52 & 326.97 & 8.491 & 1.84 & 0.59 & 12.53 & 136.17 & 1.82 & 9.10 \\
\hline $34 X-2,72-76$ & 315.12 & 329.57 & 8.611 & 1.31 & 0.71 & 4.65 & 43.30 & 1.82 & 9.10 \\
\hline $34 X-4,70-75$ & 318.10 & 332.55 & 8.787 & 2.03 & 0.60 & 9.57 & 116.62 & 1.75 & 9.16 \\
\hline $34 X-5,43-48$ & 319.33 & 333.78 & 8.844 & 1.64 & 0.43 & 24.29 & 170.91 & 1.89 & 9.05 \\
\hline $34 X-6,60-65$ & 321.00 & 335.45 & 8.945 & 1.67 & 0.77 & 16.79 & 215.51 & 1.91 & 9.03 \\
\hline $34 X-7,30-35$ & 322.20 & 336.65 & 9.005 & 2.15 & 0.74 & 10.57 & 168.44 & 1.46 & 9.42 \\
\hline $35 \mathrm{X}-1,54-58$ & 323.14 & 337.59 & 9.049 & 2.15 & 0.59 & 13.68 & 173.81 & 1.82 & 9.10 \\
\hline $35 X-2,41-46$ & 324.51 & 338.96 & 9.116 & 1.99 & 0.82 & 5.67 & 92.48 & 1.80 & 9.12 \\
\hline $35 X-3,59-64$ & 326.19 & 340.64 & 9.202 & 3.95 & 0.48 & 10.28 & 194.73 & 2.18 & 8.84 \\
\hline $35 \times-4,93-97$ & 328.03 & 342.48 & 9.293 & 1.59 & 0.68 & 1.02 & 11.09 & & \\
\hline $35 X-5,129-133$ & 329.89 & 344.34 & 9.410 & 1.62 & 0.41 & 6.83 & 45.27 & 1.80 & 9.12 \\
\hline $35 X-7,49-54$ & 332.09 & 346.54 & 9.535 & 1.94 & 0.62 & 0.79 & 9.52 & 2.06 & 8.92 \\
\hline $36 \mathrm{X}-1,60-64$ & 332.80 & 347.25 & 9.571 & 1.94 & 0.63 & 1.35 & 16.53 & & \\
\hline $36 \times-2,60-64$ & 334.30 & 348.75 & 9.690 & 1.59 & 0.65 & 7.64 & 78.97 & & \\
\hline $36 X-3,14-18$ & 335.34 & 349.79 & 9.786 & 0.99 & 0.73 & 2.16 & 15.59 & 2.05 & 8.93 \\
\hline $36 \times-5,63-67$ & 338.83 & 353.28 & 10.030 & 1.49 & 0.79 & 2.00 & 23.49 & 1.96 & 9.00 \\
\hline $36 \times-7,33-37$ & 341.53 & 355.98 & 10.212 & 1.49 & 1.01 & 10.66 & 160.17 & 1.80 & 9.12 \\
\hline
\end{tabular}

" $\mathrm{LSR}=$ linear sedimentation rate.

${ }^{\mathrm{h}} \mathrm{DBD}=$ dry-bulk density. 
Table 3. Data used to calculate the mass accumulation rate of eolian material and eolian grain-size variations in sediments from Hole 852B.

\begin{tabular}{|c|c|c|c|c|c|c|c|c|c|}
\hline $\begin{array}{l}\text { Core, section. } \\
\text { interval }(\mathrm{cm})\end{array}$ & $\begin{array}{l}\text { Depth } \\
\text { (mbsf) }\end{array}$ & $\begin{array}{l}\text { Depth } \\
\text { (med) }\end{array}$ & $\begin{array}{l}\text { Age } \\
\text { (Ma) }\end{array}$ & $\begin{array}{c}\mathrm{LSR}^{\mathrm{a}} \\
(\mathrm{cm} / \mathrm{k} \cdot \mathrm{y} .)\end{array}$ & $\begin{array}{c}\mathrm{DBD}^{b} \\
\left(\mathrm{~g} / \mathrm{cm}^{3}\right)\end{array}$ & $\begin{array}{l}\text { Eolian } \\
(\%)\end{array}$ & $\begin{array}{c}\text { Eol. flux } \\
\left(\mathrm{mg} / \mathrm{cm}^{2} \cdot \mathrm{k}_{\mathrm{y}} \mathrm{y}_{\text {. }}\right.\end{array}$ & $\begin{array}{l}\text { Mean size } \\
\qquad(\mu \mathrm{m})\end{array}$ & $\begin{array}{c}\text { Mean size } \\
\text { (ф) }\end{array}$ \\
\hline \multicolumn{10}{|l|}{$138-8.52 \mathrm{~B}-$} \\
\hline $1 \mathrm{H}-1.130-135$ & 1.30 & 1.50 & 0.116 & 1.38 & 0.51 & 16.11 & 113.72 & 1.85 & 9.08 \\
\hline $\mathrm{IH}-2.75-80$ & 2.25 & 2.45 & 0.228 & 1.00 & 0.41 & 10.14 & 41.96 & 1.89 & 9.05 \\
\hline $1 \mathrm{H}-3.140-145$ & 4.40 & 4.60 & 0.445 & 1.00 & 0.75 & 3.51 & 26.39 & 1.86 & 9.07 \\
\hline $\mathrm{IH}-5.2-7$ & 6.02 & 6.22 & 0.570 & 0.96 & 0.50 & 14.48 & 69.51 & 1.77 & 9.15 \\
\hline IH $-5.58-63$ & 6.58 & 6.78 & 0.630 & 0.58 & 0.70 & 5.11 & 20.64 & 1.85 & 9.08 \\
\hline IH-6. $35-40$ & 7.85 & 8.05 & 0.710 & 1.87 & 0.78 & 2.83 & 41.45 & 1.74 & 9.16 \\
\hline $2 \mathrm{H}-1.48-53$ & 9.38 & 9.63 & 0.833 & 1.54 & 0.41 & 20.73 & 129.73 & 1.65 & 9.24 \\
\hline $2 \mathrm{H}-1.112-117$ & 10.02 & 10.27 & 0.877 & 1.24 & 0.74 & 5.10 & 47.05 & 1.76 & 9.15 \\
\hline $2 \mathrm{H}-4,49-54$ & 13.89 & 14.14 & 1.190 & 0.72 & 0.72 & 5.12 & 26.48 & 1.75 & 9.16 \\
\hline $2 \mathrm{H}-5.85-90$ & 15.75 & 16.00 & 1.343 & 1.57 & 0.68 & 6.52 & 69.65 & 1.78 & 9.13 \\
\hline $2 \mathrm{H}-6.111-116$ & 17.51 & 17.76 & 1.483 & 1.06 & 0.42 & 20.18 & 91.17 & 1.98 & 8.98 \\
\hline $2 \mathrm{H}-7.48-53$ & 18.38 & 18.63 & 1.568 & 1.44 & 0.41 & 18.32 & 108.40 & 1.82 & 9.10 \\
\hline $3 \mathrm{H}-1,64-69$ & 19,04 & 19.84 & 1.669 & 1.09 & 0.49 & 6.75 & 36.32 & 1.81 & 9.11 \\
\hline $3 \mathrm{H}-1.113-118$ & 19.53 & 20.33 & 1.713 & 1.09 & 0.52 & 13.85 & 78.51 & 1.61 & 9.28 \\
\hline $3 \mathrm{H}-5.84-89$ & 25.24 & 26.04 & 2.281 & 1.63 & 0.72 & 9.12 & 106.66 & 1.67 & 9.22 \\
\hline $3 \mathrm{H}-6,112-117$ & 27.02 & 27.82 & 2.435 & 0.88 & 0.78 & 4.65 & 31.67 & 1.76 & 9.15 \\
\hline $3 \mathrm{H}-7.50-55$ & 27.90 & 28.70 & 2.501 & 1.40 & 0.60 & 10.97 & 92.73 & 2.00 & 8.96 \\
\hline $4 \mathrm{H}-1,17-22$ & 28.07 & 30.47 & 2.672 & 0.91 & 0.39 & 6.87 & 24.63 & 1.72 & 9.19 \\
\hline $4 \mathrm{H}-2.80-85$ & 30.20 & 32.60 & 2.834 & 0.97 & 0.76 & 6.62 & 48.78 & 1.79 & 9.13 \\
\hline $4 \mathrm{H}-3.54-59$ & 31.44 & 33.84 & 2.918 & 0.91 & 0.72 & 8.90 & 58.56 & 1.67 & 9.23 \\
\hline $4 \mathrm{H}-4,69-74$ & 33.09 & 35.49 & 3.065 & 1.29 & 0.48 & 22.49 & 139.40 & 1.75 & 9.16 \\
\hline $4 \mathrm{H}-5,124-129$ & 35.14 & 37.54 & 3.254 & 0.60 & 0.71 & 12.29 & 52.07 & 1.75 & 9.16 \\
\hline $5 \mathrm{H}-1,114-119$ & 38.54 & 41.39 & 3.639 & 0.64 & 0.30 & 35.48 & 68.78 & 1.72 & 9.18 \\
\hline $5 \mathrm{H}-3.114-119$ & 41.54 & 44.39 & 3.950 & 1.43 & 0.26 & 39.39 & 143.68 & 1.71 & 9.19 \\
\hline $5 \mathrm{H}-4,123-128$ & 43.13 & 45.98 & 4.131 & 0.86 & 0.57 & 15.91 & 78.29 & 1.87 & 9.06 \\
\hline $5 \mathrm{H}-5,114-119$ & 44.54 & 47.39 & 4.284 & 1.10 & 0.65 & 11.49 & 81.82 & 1.74 & 9.17 \\
\hline $5 \mathrm{H}-6.18-2.3$ & 45.08 & 47.93 & 4.325 & 2.70 & 0.46 & 22.40 & 277.98 & 1.77 & 9.14 \\
\hline $5 \mathrm{H}-6.142-147$ & 46.32 & 49.17 & 4.409 & 1.23 & 0.81 & 7.90 & 78.56 & 1.69 & 9.21 \\
\hline $6 \mathrm{H}-2,34-39$ & 48.74 & 53.69 & 4.745 & 3.14 & 0.75 & 4.81 & 113.01 & 1.69 & 9.21 \\
\hline $6 \mathrm{H}-2,80-85$ & 49.20 & 54.15 & 4.760 & 1.83 & 0.43 & 27.13 & 212.84 & 1.79 & 9.12 \\
\hline $6 \mathrm{H}-3,142-147$ & 51.32 & 56.27 & 4.911 & 1.22 & 0.71 & 8.36 & 72.71 & 1.74 & 9.17 \\
\hline $6 \mathrm{H}-5,50-55$ & 53.40 & 58.35 & 5.042 & 1.66 & 0.68 & 8.68 & 98.04 & 1.77 & 9.14 \\
\hline $6 \mathrm{H}-6.60-65$ & 55.00 & 59.95 & 5.123 & 2.41 & 0.81 & 3.87 & 75.77 & 1.89 & 9.05 \\
\hline $6 \mathrm{H}-7,13-18$ & 56.03 & 60.98 & 5.175 & 1.07 & 0.6 .3 & 15.19 & 103,06 & 1.81 & 9.11 \\
\hline $7 \mathrm{H}-1,36-41$ & 56.76 & 62.61 & 5.288 & 1.89 & 0.76 & 4.27 & 61.70 & 1.79 & 9.13 \\
\hline $7 \mathrm{H}-1.115-120$ & 57.55 & 63.40 & 5.327 & 2.55 & 0.55 & 18.28 & 257,30 & 1.79 & 9.13 \\
\hline $7 \mathrm{H}-2,101-106$ & 58.69 & 64.54 & 5.403 & 1.86 & 0.50 & 17.50 & 163.98 & 1.71 & 9.19 \\
\hline $7 \mathrm{H}-5.48-53$ & 62.66 & 68.51 & 5.674 & 1.30 & 0.80 & 3.01 & 31.32 & 1.90 & 9.04 \\
\hline $7 \mathrm{H}-5,145-150$ & 63.63 & 69.48 & 5.729 & 1.64 & 0.48 & 28.97 & 226.23 & 1.82 & 9.11 \\
\hline $7 \mathrm{H}-7.70-75$ & 65.88 & 71.73 & 5.867 & 1.60 & 0.81 & 5.15 & 67.04 & 1.81 & 9.11 \\
\hline $8 \mathrm{H}-1,5 \mathrm{I}-56$ & 66.41 & 73.16 & 5.960 & 2.07 & 0,80 & 4.02 & 66.86 & 1.76 & 9.15 \\
\hline $8 \mathrm{H}-2,1,36-14 \mathrm{I}$ & 68.76 & 75.51 & 6.066 & 2.20 & 0.68 & 10.51 & 157.58 & 1.78 & 9.14 \\
\hline $8 \mathrm{H}-3,3 \mathrm{I}-36$ & 69.21 & 75.96 & 6.094 & 1.10 & 0.80 & 3.88 & 34.16 & 1.74 & 9.17 \\
\hline $8 \mathrm{H}-4,141-146$ & 71.81 & 78.56 & 6.233 & 1.83 & 0.69 & 7.62 & 96.44 & 1.84 & 9.09 \\
\hline $8 \mathrm{H}-5.50-55$ & 72.40 & 79.15 & 6.269 & 1.60 & 0.66 & 10.90 & 115.94 & 1.78 & 9.14 \\
\hline $8 \mathrm{H}-6,113-118$ & 74.53 & 81.28 & 6.444 & 1.08 & 0.79 & 6.36 & 54,02 & 1.77 & 9.14 \\
\hline $9 \mathrm{H}-1.60-65$ & 76.00 & 84.50 & 6.677 & 1.10 & 0.68 & 7.04 & 52.72 & 1.91 & 9.03 \\
\hline $9 \mathrm{H}-2,60-65$ & 77.50 & 86.00 & 6.775 & 2.42 & 0.82 & 3.74 & 74.56 & 1.95 & 9.00 \\
\hline $9 \mathrm{H}-3,60-65$ & 79.00 & 87.50 & 6.837 & 2.42 & 0.83 & 4.25 & 85.18 & 2.09 & 8.90 \\
\hline $9 \mathrm{H}-4,60-65$ & 80.50 & 89.00 & 6.899 & 2.42 & 0.84 & 2.57 & 52.33 & 1.90 & 9.04 \\
\hline $9 \mathrm{H}-5,60-65$ & 82.00 & 90.50 & 6.988 & 1.21 & 0.79 & 5.07 & 48.25 & 1.88 & 9.05 \\
\hline $9 \mathrm{H}-6.60-65$ & 83.50 & 92.00 & 7.112 & 1.21 & 0.79 & 4.05 & 38.63 & 2.01 & 8.96 \\
\hline $9 \mathrm{H}-7.10-15$ & 84.50 & 93.00 & 7.195 & 1.21 & 0.73 & 5.97 & 52.55 & 2.11 & 8.89 \\
\hline $10 \mathrm{H}-\mathrm{I} .58-63$ & 85.48 & 95.48 & 7.400 & 1.21 & 0.79 & 5.57 & 53.54 & & \\
\hline $10 \mathrm{H}-2.56-61$ & 86.96 & 96.96 & 7.536 & 1.24 & 0.77 & 4.65 & 44.57 & 1.95 & 9.00 \\
\hline $10 \mathrm{H}-3.58-63$ & 88.48 & 98.48 & 7.659 & 1.24 & 0.62 & 10.16 & 78.08 & & \\
\hline $\mathrm{IOH}-4.53-58$ & 89.93 & 99.93 & 7.762 & 2.00 & 0.84 & 3.49 & 58.39 & 2.00 & 8.97 \\
\hline $10 \mathrm{H}-5.58-63$ & 91.48 & 101.48 & 7.842 & 1.79 & 0.73 & 6.62 & 86.68 & 2.03 & 8.95 \\
\hline $10 \mathrm{H}-6.58-63$ & 92.98 & 102.98 & 7.914 & 2.64 & 0.89 & 2.78 & 65.44 & 1.91 & 9.03 \\
\hline $10 \mathrm{H}-7.58-63$ & 94.48 & 104.48 & 7.998 & 1.86 & 0.93 & 4.33 & 74.91 & 1.88 & 9.06 \\
\hline $\mathrm{IIH}-1.50-55$ & 94.90 & 105.90 & 8.066 & 2.26 & 0.82 & 5.16 & 95.00 & 1.89 & 9.05 \\
\hline $\mathrm{IIH}-2.60-65$ & 96.50 & 107.50 & 8.137 & 2.26 & 0.83 & 3.60 & 67.38 & & \\
\hline $11 \mathrm{H}-3.60-65$ & 98.00 & 109.00 & 8.226 & 1.31 & 0.82 & 3.30 & 35.25 & 1.99 & 8.97 \\
\hline IIH-4.60-65 & 99.50 & 110.50 & 8.340 & 1.31 & 0.96 & 4.63 & 58.01 & & \\
\hline IIH-5. 60-65 & 101.00 & 112.00 & 8.455 & $|3|$ & 0.97 & 2.27 & 28.99 & 1.87 & 9.07 \\
\hline IIH-6. $60-65$ & 102.50 & 113.50 & 8.569 & 1.31 & 0.88 & 5.78 & 66.55 & 2.07 & 8.92 \\
\hline $12 \mathrm{H}-1.60-64$ & 104.40 & 116.60 & 8.945 & 0.36 & 0.51 & 18.47 & 33.75 & 2.09 & \\
\hline $12 \mathrm{H}-2.60-64$ & 106.00 & 118.20 & 9.246 & 0.36 & 0.43 & 27.52 & 42.10 & 1.95 & 9.00 \\
\hline $12 \mathrm{H}-3.60-64$ & 107.50 & 119.70 & 9.651 & 0.51 & 0.46 & 28.36 & 66.83 & 2.03 & \\
\hline $12 \mathrm{H}-4,60-64$ & 109.00 & 121.20 & 9.891 & 0.92 & 0.51 & 18.72 & 88.05 & 2.02 & 8.95 \\
\hline $12 \mathrm{H}-5.60-64$ & 110.50 & 122.70 & 10.052 & 0.99 & 0.66 & 8.39 & 54.53 & 1.92 & 9.03 \\
\hline $12 \mathrm{H}-6.60-64$ & 112.00 & 124.20 & 10.204 & 0.99 & 0.49 & 35.85 & 173.51 & 1.98 & 8.98 \\
\hline $12 \mathrm{H}-7.60-64$ & $11,3,50$ & 125.70 & 10.356 & 0.99 & 0.47 & 49.19 & 229.50 & 1.89 & 9.05 \\
\hline
\end{tabular}

a $\mathrm{LSR}=$ linear sedimentation rate.

${ }^{\mathrm{b}} \mathrm{DBD}=$ dry-bulk density. 
Table 4. Data used to calculate the mass accumulation rate of eolian material and eolian grain-size variations in sediments from Hole 853B.

\begin{tabular}{|c|c|c|c|c|c|c|c|c|c|}
\hline $\begin{array}{l}\text { Core, section. } \\
\text { interval }(\mathrm{cm})\end{array}$ & $\begin{array}{l}\text { Depth } \\
\text { (mbsf) }\end{array}$ & $\begin{array}{l}\text { Depth } \\
\text { (mcd) }\end{array}$ & $\begin{array}{l}\text { Age } \\
(\mathrm{Ma})\end{array}$ & $\begin{array}{l}\mathrm{LSR}^{\mathrm{A}} \\
(\mathrm{cm} / \mathrm{k} \cdot \mathrm{y} .)\end{array}$ & $\begin{array}{l}\text { DBDb } \\
\left(\mathrm{g} / \mathrm{cm}^{3}\right)\end{array}$ & $\begin{array}{l}\text { Eolian } \\
(\%)\end{array}$ & $\begin{array}{c}\text { Eol. flux } \\
\left(\mathrm{mg} / \mathrm{cm}^{2} \bullet \mathrm{k} . \mathrm{y} .\right)\end{array}$ & $\begin{array}{l}\text { Mean size } \\
\qquad(\mu \mathrm{m})\end{array}$ & $\begin{array}{c}\text { Mean size } \\
\text { (ф) }\end{array}$ \\
\hline \multicolumn{10}{|l|}{ 138-853B- } \\
\hline IH-I, 60-64 & 0.60 & 0.60 & 0.116 & 0.33 & 0.46 & 12.61 & 19.14 & 1.96 & 8.99 \\
\hline $\mathrm{IH}-2,60-64$ & 2.10 & 2.10 & 0.571 & 0.34 & 0.59 & 10.69 & 21.44 & 2.18 & 8.84 \\
\hline $1 \mathrm{H}-3,60-64$ & 3.60 & 3.60 & 0.932 & 0.43 & 0.45 & 18.05 & 34.93 & 2.03 & 8.94 \\
\hline $2 \mathrm{H}-\mathrm{I}, 60-64$ & 4.90 & 4.85 & 1.198 & 0.52 & 0.48 & 13.34 & 33.30 & 1.79 & 9.13 \\
\hline $2 \mathrm{H}-2,60-64$ & 6.40 & 6.35 & 1.489 & 0.52 & 0.44 & 20.98 & 48.00 & 1.95 & 9.00 \\
\hline $2 \mathrm{H}-3,60-64$ & 7.90 & 7.85 & 1.782 & 0.43 & 0.52 & 16.79 & 37.54 & 1.90 & 9.04 \\
\hline $2 \mathrm{H}-4,60-64$ & 9.40 & 9.35 & 2.090 & 0.56 & 0.76 & 9.42 & 40.09 & 1.85 & 9.08 \\
\hline $2 \mathrm{H}-5,60-64$ & 10.90 & 10.85 & 2.361 & 0.56 & 0.76 & 6.39 & 27.20 & 1.88 & 9.05 \\
\hline $2 \mathrm{H}-6,60-64$ & 12.40 & 12.35 & 2.625 & 0.67 & 0.62 & 9.12 & 37.88 & 1.77 & 9.14 \\
\hline $2 \mathrm{H}-7,60-64$ & 13.90 & 13.85 & 2.849 & 0.67 & 0.63 & 12.16 & 51.33 & 1.95 & 9.00 \\
\hline $3 \mathrm{H}-1,55-60$ & 12.85 & 15.25 & 3.057 & 0.70 & 0.49 & 26.61 & 91.27 & 2.11 & 8.89 \\
\hline $3 \mathrm{H}-2,55-60$ & 14.35 & 16.75 & 3.299 & 0.54 & 0.60 & 17.94 & 58.13 & 1.77 & 9.14 \\
\hline $3 \mathrm{H}-3,55-60$ & 15.85 & 18.25 & 3.527 & 0.67 & 0.49 & 16.96 & 55,68 & 2.03 & 8.95 \\
\hline $3 \mathrm{H}-4,55-60$ & 17.35 & 19.75 & 3.766 & 0.60 & 0,48 & 25.30 & 72.86 & 1.89 & 9.05 \\
\hline $3 \mathrm{H}-5,55-60$ & 18.85 & 21.25 & 4.014 & 0.60 & 0.45 & 30.28 & 81.76 & 1.75 & 9.16 \\
\hline $3 \mathrm{H}-6,55-60$ & 20.35 & 22.75 & 4.236 & 0.93 & 0.53 & 20.64 & 74.33 & 1,84 & 9.05 \\
\hline $4 \mathrm{H}-1,55-60$ & 20.85 & 21.85 & 4.114 & 0.60 & 0.54 & 14.80 & 65.64 & 1.89 & 9.08 \\
\hline $4 \mathrm{H}-2,55-60$ & 22.35 & 23.35 & 4.301 & 0.93 & 0.56 & 17.44 & 90.83 & 1.66 & 9.23 \\
\hline $4 \mathrm{H}-3,55-60$ & 23.85 & 24.85 & 4.422 & 1.30 & 0.55 & 22.28 & 159.30 & 1.80 & 9.12 \\
\hline $4 \mathrm{H}-4,55-60$ & 25.35 & 26.35 & 4.658 & 0.74 & 0.44 & 23.62 & 76.91 & 1.77 & 9.14 \\
\hline $4 \mathrm{H}-5,55-60$ & 26.85 & 27.85 & 4.851 & 0.88 & 0.66 & 13.23 & 76.84 & 1.79 & 9.12 \\
\hline $4 \mathrm{H}-6,55-60$ & 28.35 & 29.35 & 5.040 & 1.08 & 0.78 & 8.53 & 71.86 & 1.75 & 9.16 \\
\hline $5 \mathrm{H}-1,55-60$ & 28.85 & 29.85 & 5.087 & 1.08 & 0.54 & 20.40 & 118.97 & 1.77 & 9.14 \\
\hline $5 \mathrm{H}-2,55-60$ & 30.35 & 31.35 & 5.226 & 1.08 & 0.60 & 14.42 & 93.44 & 1.70 & 9.20 \\
\hline $5 \mathrm{H}-3,96-101$ & 32.26 & 33.26 & 5.398 & 1.11 & 0.74 & 5.63 & 46.24 & 1.78 & 9.13 \\
\hline $5 \mathrm{H}-4,8-13$ & 32.88 & 33.88 & 5.454 & 1.11 & 0.53 & 21.51 & 126.54 & 1.59 & 9.29 \\
\hline $5 \mathrm{H}-4,143-148$ & 34.23 & 35.23 & 5.575 & 1.11 & 0.44 & 28.93 & 141.29 & 1.69 & 9.21 \\
\hline $5 \mathrm{H}-6,55-60$ & 36.35 & 37.35 & 5.765 & 1.11 & 0.71 & 10.14 & 79.91 & 1.70 & 9.20 \\
\hline $5 \mathrm{H}-7,64-69$ & 37.84 & 38.84 & 5.896 & 1.31 & 0.64 & 14.59 & 122.32 & 1.72 & 9.19 \\
\hline $6 \mathrm{H}-1,59-63$ & 38.39 & 39.39 & 5.938 & 1.31 & 0.73 & 8.72 & 83.39 & 1.88 & 9.05 \\
\hline $6 \mathrm{H}-2,42-47$ & 39.72 & 40.72 & 6.039 & 1.31 & 0.61 & 16.57 & 132.41 & 1.86 & 9.07 \\
\hline $6 \mathrm{H}-3,83-88$ & 41.63 & 42.63 & 6.186 & 1.31 & 0.69 & 12.02 & 108.65 & 1.86 & 9.07 \\
\hline $6 \mathrm{H}-4,0-5$ & 42.30 & 43.30 & 6.237 & 1.31 & 0.56 & 20.55 & 150.75 & 1.82 & 9.10 \\
\hline $6 \mathrm{H}-5,94-97$ & 44.74 & 45.74 & 6.476 & 1.00 & 0.79 & 10.97 & 86.66 & 1.81 & 9.11 \\
\hline $6 \mathrm{H}-6,18-23$ & 45.48 & 46.48 & 6.550 & 1.00 & 0.75 & 8.62 & 64.65 & 1.65 & 9.25 \\
\hline $6 \mathrm{H}-7,54-59$ & 47.34 & 48.34 & 6.677 & 1.47 & 0.75 & 7.53 & 83.02 & 1.81 & 9.11 \\
\hline $7 \mathrm{H}-1,60-65$ & 47.90 & 48.90 & 6.715 & 1.47 & 0.80 & 6.85 & 80.56 & 1.80 & 9.12 \\
\hline $7 \mathrm{H}-2,140-145$ & 50.20 & 51.20 & 6.871 & 1.47 & 0.53 & 24.80 & 193.22 & 1.86 & 9.07 \\
\hline $7 \mathrm{H}-3,85-90$ & 51.15 & 52.15 & 6.936 & 1.47 & 0.60 & 24.85 & 219.18 & 1.82 & 9.10 \\
\hline $7 \mathrm{H}-4,60-65$ & 52.40 & 53.40 & 7.020 & 1.47 & 0.65 & 15.51 & 148.20 & 1.69 & 9.21 \\
\hline $7 \mathrm{H}-5,7-12$ & 53.37 & 54.37 & 7.109 & 0.57 & 0.77 & 8.79 & 38.58 & 1.86 & 9.07 \\
\hline $7 \mathrm{H}-6,92-97$ & 55.72 & 56.72 & 7.418 & 1.38 & 0.51 & 36.49 & 256.82 & 2.08 & 8.91 \\
\hline $7 \mathrm{H}-7,64-69$ & 56.94 & 57.94 & 7.507 & 1.38 & 0.70 & 10.80 & 104.33 & 1.71 & 9.19 \\
\hline $8 \mathrm{H}-\mathrm{I}, 79-84$ & 57.59 & 58.59 & 7.566 & 0.87 & 0.79 & 4.97 & 34.16 & 1.71 & 9.20 \\
\hline $8 \mathrm{H}-2,80-85$ & 59.10 & 60.10 & 7.684 & 1.60 & 0.66 & 13.83 & 146.04 & 1.75 & 9.16 \\
\hline $8 \mathrm{H}-3,60-65$ & 60.40 & 61.40 & 7.765 & 1.60 & 0.73 & 8.81 & 102.90 & 1.82 & 9.11 \\
\hline $8 \mathrm{H}-4,134-139$ & 62.64 & 63.64 & 7.905 & 1.60 & 0.82 & 9.03 & 118.47 & 1.71 & 9.19 \\
\hline $8 \mathrm{H}-5,78-83$ & 63.58 & 64.58 & 7.963 & 1.60 & 0.69 & 21.28 & 234.93 & 1.73 & 9.18 \\
\hline $8 \mathrm{H}-6,57-62$ & 64.87 & 65.87 & 8.041 & 1.92 & 0.78 & 11.71 & 175.37 & 1.68 & 9.22 \\
\hline $8 \mathrm{H}-7,48-53$ & 66.28 & 67.28 & 8.115 & 1.92 & 0.85 & 9.98 & 162.87 & 1.77 & 9.14 \\
\hline $9 \mathrm{H}-\mathrm{I}, 80-85$ & 67.10 & 68.10 & 8.157 & 1.92 & 0.73 & 28.12 & 394.13 & 2.01 & 8.96 \\
\hline $9 \mathrm{H}-2,80-85$ & 68.60 & 69.60 & 8.248 & 1.41 & 0.58 & 26.97 & 220.56 & 1.64 & 9.25 \\
\hline $9 \mathrm{H}-3,10-15$ & 69.40 & 70.40 & 8.304 & 1.41 & 0.58 & 27.17 & 222.20 & 1.96 & 9.00 \\
\hline $9 \mathrm{H}-4,75-80$ & 71.36 & 72.36 & 8.443 & 1.41 & 0.36 & 22.28 & 113.09 & 1.82 & 9.10 \\
\hline $9 \mathrm{H}-5,26-30$ & 71.83 & 72.83 & 8.477 & 0.58 & 0.46 & 33.08 & 88.26 & 1.80 & 9.12 \\
\hline
\end{tabular}

ITCZ through the geologic past. Eolian records from Leg 138, therefore, not only provide a history of atmospheric circulation asymmetry, but also the opportunity to examine the long-term relationship and interaction between atmospheric and oceanic circulation in this region.

The grain-size of eolian sediments from Site 849 are consistently offset smaller by about 0.10 to $0.15 \phi$ than those from Site 848 . The explanation for this lies in the modern pattern of atmospheric circulation intensity, which is weaker in the ITCZ and increases as the trade winds are approached to the north and south (Fig. 4). The fact that this offset is recorded over the entire interval suggests that the ITCZ remained north of Sites 848 and 849 throughout the late Neogene. Temporal variations in eolian grain-sizes to these sites, therefore, provide a history of southern trade-wind intensity. Over the past $8.0 \mathrm{Ma}$, the coarsest eolian material was deposited in the late Miocene (about 8.0 to $5.0 \mathrm{Ma}$ ) implying that more intense southern trade winds occurred at this time. As expected, higher sedimentation rates related to increased biological productivity are also recorded in equatorial sedi- ments during this interval (Fig. 7). Eolian grain-sizes gradually decreased during the early Pliocene (about 5.0 to $4.0 \mathrm{Ma}$ ) and, except for a brief coarser interval near about $3.0 \mathrm{Ma}$, remained relatively constant in the younger portion. Again, a decline in sedimentation rates occurred along the equator beginning at the same time southeast trade-wind intensity decreased strongly, suggesting a close relationship between atmospheric circulation strength, equatorial upwelling, and regional productivity. The pattern of eolian size variability from Site 852 is broadly similar to the southern sites (Figs. 7 and 8 ). However, because this site is located much closer to the axis of the ITCZ, the eolian grain-size record is characterized by much finer mean sizes and less variability than at Sites 848 and 849 . The record from Site 853 shows a distinctly different pattern than the others and shows finer eolian material was transported to this site in the older portion of the record while the younger portion is characterized by two intervals of coarsening, one in the early Pliocene (from about 4.0 to $3.0 \mathrm{Ma}$ ) and another in the Pleistocene, beginning about 1.0 Ma. The first interval of coars- 
ening reflects a shift in the latitudinal position of the ITCZ (see discussion below). Although no response to the onset of Northern Hemisphere glaciation is apparent from these data, the coarsening of eolian sizes at 1.0 Ma suggests that northern trade winds may have intensified at this time, possibly in response to the mid-Pleistocene increase in the amplitude of glacial-interglacial oscillations (Pisias and Moore, 1981).

Eolian records determined for this study also suggest that increased terrigenous deposition rates occur beneath the ITCZ; average Pleistocene values are greatest at Site 852 and decrease by more than $50 \%$ to the north and south (Fig. 8). On a regional scale, the overall average flux of eolian material has decreased throughout the late Neogene (Fig. 8). Much of this decline can be related to a reduction in eolian input to the northern sites (Sites 852 and 853) during the late Pliocene. The supply of eolian material from continental source regions is closely related to the climate; arid climate conditions supply relatively more dust material than wetter, more humid conditions (Pye, 1987). Thus, the decline in eolian accumulation rates in the equatorial Pacific reflects regionally wetter conditions in central and northern South America at this time.

Because variations in both the accumulation rate and mean grainsize of eolian materials are related to the position of the ITCZ, temporal changes in the regional pattern of these records reflect latitudinal movement of the ITCZ throughout the geologic past. Eolian grainsize data show that the modern pattern eolian deposition has continued at least since the early Pliocene (Fig. 8). Prior to about $4 \mathrm{Ma}$, however, the position of minimum eolian grain-size deposition shifted northward and the component of northern coarsening is lost from these records, suggesting that the ITCZ was located north of Site 853 (about $7^{\circ} \mathrm{N}$ ) during the late Miocene. Eolian flux data also record a similar history of ITCZ movement, indicating maximum eolian accumulation rates located farther north than at present.

\section{SUMMARY}

Temporal and latitudinal patterns of eolian accumulation and grainsize variability provide important information about the strength and position of equatorial trade-wind circulation throughout the late Neogene. Eolian sediments from Sites 848 and 849 provide a history of southern atmospheric circulation intensity and suggest the strongest southeast trade winds occurred during the late Miocene, from about 8 to $5 \mathrm{Ma}$. Higher rates of biogenic sedimentation also occur along the equator during this interval and may be associated with increased divergent upwelling caused by more intense southeast trade-wind circulation. Trade-wind strength decreased from about 5.0 to $4.0 \mathrm{Ma}$ and, except for a brief interval near $3.0 \mathrm{Ma}$, has remained relatively constant since then. No apparent response to the onset of Northern Hemisphere glaciation is recorded by the size of eolian material transported by southeast trade winds. Northeast trade-wind intensity is recorded by eolian sediments spanning the past 4 m.y. of deposition at Site 853. These sediments suggest that an increase in the intensity of northeast trade winds occurred at about $1.0 \mathrm{Ma}$ but no significant change is associated with the onset of Northern Hemisphere glaciation.

The accumulation rate of eolian material suggests a regional decrease in dust input to the ocean occurred during the late Pliocene. This decrease reflects a diminished supply of eolian material from the continental source region, which may be related to an increase in the wetness of Central and South American climates at this time.

Regional patterns of eolian deposition are associated with the ITCZ and can be used to track its latitudinal position in the past. Eolian grain-size variability and eolian flux records both suggest that prior to about 4.0 to $5.0 \mathrm{Ma}$ the ITCZ was located north of Site $853\left(7^{\circ} \mathrm{N}\right.$ paleolatitude) and began moving southward to its present location near the end of the late Miocene.

\section{ACKNOWLEDGMENTS}

I am grateful to Dave Rea and Ted Moore for many insightful ideas and discussions regarding this project. I also thank S. Clemens, F.
Sirocko, and T.H. van Andel for helpful reviews and suggestions concerning this manuscript. Carol Copeland, Tooba Durrani, Cindi Erickson, and Pamela Warner were especially helpful with laboratory analysis. This work was supported by grants from JOI-USSAC ODP Fellowship (JSC-CY6-2) and the U.S. Science Support Program.

\section{REFERENCES}

Boyce, R.E., 1976. Definitions and laboratory techniques of compressional sound velocity parameters and wet-water content, wet-bulk density, and porosity parameters by gravimetric and gamma ray attenuation techniques. In Schlanger, S.O., Jackson, E.D., et al., Init. Repts. DSDP, 33: Washington (U.S. Govt. Printing Office), 931-958.

Chuey, J.M., Rea, D.K., and Pisias, N.G., 1987. Late Pleistocene paleoclimatology of the central Equatorial Pacific: a quantitative record of eolian and carbonate deposition. Quat. Res., 28:323-339.

Clemens, S.C., and Prell, W.L., 1990. Late Pleistocene variability of Arabian Sea summer monsoon winds and continental aridity: eolian records from the lithogenic component of deep-sea sediments. Paleoceanography, 5:109-145.

Cox, A., and Engebretson, D., 1985. Changes in motion of the Pacific plate at 5 Myr BP. Nature, 313:472-474.

Flohn, H., 1981. A hemispheric circulation asymmetry during late Tertiary. Geol. Rundsch., 70:725-736.

Gillette, D.A., Blifford, I.H., Jr., and Fryrear, D.W., 1974. The influence of wind velocity on the size distributions of aerosols generated by wind erosion of soils. Geophys. Res., 79:295-321.

Glaccum, R.A., and Prospero, J.M., 1980. Saharan aerosols over the tropical North Atlantic-mineralogy. Mar. Geol., 37:295-321.

Jaenicke, R., 1979. Monitoring and critical review of the estimates source strength of mineral dust from the Sahara. In Morales, C. (Ed.), Saharan Dust: Mobilization, Transport and Deposition: New York (Wiley), 233-242.

Janecek, T.R., 1985. Eolian sedimentation in the Northwest Pacific Ocean: a preliminary examination of the data from Deep Sea Drilling Project Sites 576 and 578. In Heath, G.R., Burckle, L.H., et al., Init. Repts. DSDP, 86: Washington (U.S. Govt. Printing Office), 589-603.

Janecek, T.R., and Rea, D.K., 1985. Quaternary fluctuation in the Northern Hemisphere tradewinds and westeries. Quat. Res., 24:150-163.

Lyle, M., 1992. Composition maps of surface sediments of the eastern tropical Pacific Ocean. In Mayer, L., Pisias, N., Janecek, T., et al., Proc. ODP, Init. Repts., 138 (Pt. 1): College Station, TX(Ocean Drilling Program), 101-115.

Mayer, L., Pisias, N., Janecek, T., et al., 1992. Proc. ODP, Init. Repts., 138 (Pts. 1 and 2): College Station, TX (Ocean Drilling Program).

Nakai, S., Halliday, A.N., and Rea, D.K., in press. Provenance of dust in the Pacific Ocean. Earth Planet. Sci. Lett.

Pisias, N., and Rea, D.K., 1988. Late Pleistocene paleoclimatology of the central equatorial Pacific: sea surface response to the southeast trade winds. Paleoceanography, 3:21-37.

Pisias, N.G., and Moore, T.C., Jr., 1981. The evolution of Pleistocene climate: a time series approach. Earth Planet. Sci. Lett., 52:450-458.

Prospero, J.M., 1981. Arid regions as sources of mineral aerosols in the marine atmosphere. In Pewe, T.L. (Ed.), Desert Dust: Origin, Characteristics, and Effects on Man. Spec. Pap.-Geol. Soc. Am., 186:71-86.

Prospero, J.M., and Nees, R.T., 1986. Impact of the North African drought and El Niño on mineral dust in the Barbados trade winds. Nature, 320:735-738.

Pye, K., 1987. Aeolian Dust and Dust Deposits: London (Academic Press).

Raemdonck, H., Maenhout, W., and Andreae, M.O., 1986. Chemistry of marine aerosol over the tropical and equatorial Pacific. J. Geophys. Res., 91:8623-8636.

Ramage, C.S., Khalsa, S.J.S., and Meisner, B.N., 1981. The central Pacific near-equatorial convergence zone. J. Geophys. Res., 86:6580-6598.

Rea, D.K., 1990. Aspects of atmospheric circulation: the late Pleistocene $(0-950,000 \mathrm{yr})$ record of eolian deposition in the Pacific Ocean. Palaeogeogr., Palaeoclimatol., Palaeoecol., 78:217-227.

Rea, D.K., and Janecek, T.R., 1981. Mass-accumulation rates of the nonauthigenic inorganic crystalline (eolian) component of deep-sea sediments from the western mid-Pacific Mountains, Deep Sea Drilling Project Site 463. In Theide, J., Vallier, T.L., et al., Init. Repts. DSDP, 62: Washington (U.S. Govt. Printing Office), 653-659.

\footnotetext{
Abbreviations for names of organizations and publication titles in ODP reference lists follow the style given in Chemical Abstracts Service Source Index (published by American Chemical Society).
} 
Rea, D.K., Leinen, M., and Janecek, T.R., 1985. A geological approach to the long-term history of atmospheric circulation. Science, 227:721-725.

Rea, D.K., Pisias, N.G., and Newberry, T., 1991. Late Pleistocene paleoclimatology of the central Equatorial Pacific: flux patterns of biogenic sediments. Paleoceanography, 6:227-244.

Schutz, L., Jaenicke, R., and Pietrek, H., 1981. Saharan dust transport over the North Atlantic Ocean. In Pewe, T.L. (Ed.), Desert Dust: Origin, Charac. teristics and Effect on Man. Spec. Pap.-Geol. Soc. Am., 186:87-100.

Windom, H.L., 1969. Atmospheric dust records in permanent snowfields: implications to marine sedimentation. Geol. Soc. Am. Bull., 80:761-782.
Wyrtki, K., 1974. Equatorial currents in the Pacific 1950-1970 and their relations to Trade Winds. J. Phys. Oceanogr., 4:372-380.

Wyrtki, K., and Kilonsky, B., 1984. Mean water and current structure during the Hawaii-to-Tahiti shuttle experiment. J. Phys. Oceanogr., 14:242-254.

Date of initial receipt: 1 February 1993

Date of acceptance: 26 July 1993

Ms 138SR-132 